\title{
Coordinated SLNR based Precoding in Large-Scale Heterogeneous Networks
}

\author{
Ikram Boukhedimi, Abla Kammoun, and Mohamed-Slim Alouini \\ Computer, Electrical, and Mathematical Sciences and Engineering (CEMSE) Division, \\ King Abdullah University of Science and Technology (KAUST), \\ Thuwal, Makkah Province, Kingdom of Saudi Arabia. \\ Email: $\{$ ikram.boukhedimi, abla.kammoun, slim.alouini $\} @$ kaust.edu.sa
}

\begin{abstract}
This work focuses on the downlink of large-scale two-tier heterogeneous networks composed of a macro-cell overlaid by micro-cell networks. Our interest is on the design of coordinated beamforming techniques that allow to mitigate the inter-cell interference. Particularly, we consider the case in which the coordinating base stations (BSs) have imperfect knowledge of the channel state information. Under this setting, we propose a regularized SLNR based precoding design in which the regularization factor is used to allow better resilience with respect to the channel estimation errors. Based on tools from random matrix theory, we provide an analytical analysis of the SINR and SLNR performances. These results are then exploited to propose a proper setting of the regularization factor. Simulation results are finally provided in order to validate our findings and to confirm the performance of the proposed precoding scheme.
\end{abstract}

Index Terms-Coordinated Beamforming, Heterogeneous Networks, Large-scale MIMO systems, Random Matrix Theory, Signal-to-Leakage Beamforming, Optimal precoding.

\section{INTRODUCTION}

With the ever-increasing demand of mobile data traffic, future $5 \mathrm{G}$ systems are required to support higher data rates and wider coverages [1]- [4]. Obviously, these requirements cannot be accommodated by current technologies. There is today a general consensus that responding to the challenges of future 5G systems can only be attained by network densification. Massive MIMO systems and micro-cells are the two ostensibly different technologies that have been proposed towards achieving network densification. The former aims to ensure a wide coverage and to support high-mobility by equipping macro-cell base stations (BSs) with very large antenna arrays. On the other hand, the latter relies on a dense deployment of low-cost and low-power micro-cells [5], [6], allowing to bring the user equipment (UE) and its serving station close to one another. This has been shown to enhance indoor coverage and improve the cell-edge performances [7], [8]. However, each technology alone could not rise to the big challenges of $5 \mathrm{G}$ future generation systems. An important research effort is now being carried out to develop signal processing methods that facilitate the co-existence and interplay between both types of networks. The resulting network architecture is often termed as two-tier heterogeneous networks (HetNet) in reference to their possessing different characteristics. Of major importance in practice is the management of the interference between the two tiers [9]. If not properly handled, it is well-recognized that this interference may lead to significant performance losses.
To overcome the interference problem, coordinated beamforming based techniques have received a lot of interest [10][14]. The main idea is to use precoding schemes that exploit information about the interference that each BS causes to the neighboring cells [15]. In TDD systems, the coordinating BSs will acquire CSI by means of pilot sequences sent from UEs to their serving BS. This is arguably less demanding than joint processing techniques (see [16] and the references therein), which requires BSs to share both CSI and data

. Usually, the design of coordinated beamforming is based on one of the following criteria. One can consider the minimization of the transmit power under some target constraints on the signal to noise ratio (SINR) [17] or the complete elimination of the interference through the use of Zero-Forcing beamforming (ZFBF), [18]. The design criterion that is considered in this work is the Signal-to-Leakage-and-Noise ratio (SLNR), which uses the concept of leakage. This latter refers to the amount of interference caused by the signal intended for the UE of interest on the remaining users; in contrast to conventional SINR based schemes which involves the interference suffered by the desired UE.

The main literature related to the use of the SLNR for the precoding design is represented by [19]-[23]. However, most of these works consider single-cell multi-user systems or noncoordinated scenarios and focus on the perfect CSI case. Under these settings, it is already known in [19] that the resulting beamforming that maximizes the SLNR coincides with wellknown transmit Wiener filter [18]. In [24], the authors carry out a simulation-based analysis of coordinated SLNR based precoding in HetNets. To the best of our knowledge, theoretical analysis of the design of SLNR based precoding for HetNets and assuming imperfect CSI have never been conducted. This constitutes the focus of our paper. Particularly, we consider a HetNet in which each BS employs a regularized variant of the classical SLNR based precoding, which we refer to as SLNR-MAX precoding. The novelty of our proposed scheme lies in the use of a regularization parameter that weights the other cells information used in the precoding. Intuitively, the setting of this weight should depend on the reliability of the available CSI, taking higher values for good CSI qualities and lower values in the opposite case. A key question in this respect is how to select a proper value for this regularization factor. To tackle this issue, we consider the asymptotic regime in which the number of UEs per cell and the number of antennas 
deployed in each BS increase with the same pace, which allows us to leverage results from random matrix theory [25]-[27]. The analysis provides accurate closed-form approximations for the SINR and SLNR for each BS and UE pair that depend solely on the channel large-scale statistics and the power of the channel estimation error. The results concerning the SLNR are then optimized in order to get a proper selection method for the regularization parameter.

The remainder of the paper is organized as follows. In the next section, we introduce the system model and review the SLNR based precoding schemes. In Section III, we present our main results analyzing SINR and SLNR when using the proposed SLNR-MAX precoding. These results lead us to propose a proper way to select the regularization parameter in III-C. Section IV provides a selection of numerical illustrations that validate our theoretical findings and shed light on some interesting aspects. Finally, conclusions are drawn in Section V.

\section{Notations}

In the sequel, bold upper and lowercase characters refer to matrices and vectors, respectively. We use $(.)^{\mathrm{H}}, \operatorname{tr}($.$) and (.)^{-1}$ to denote the conjugate transpose, the trace of a matrix and the inverse operations, respectively. $\log ($.$) is the natural logarithm$ and the $N \times N$ identity matrix is denoted $\mathbf{I}_{N}$. $\mathbb{E}[$.] represents the statistical expectation.

\section{SySTEM MOdEL}

In this section we introduce the transmission system, the considered channel model, and the coordinated beamforming design.

\section{A. Transmission Model}

We consider the downlink of a TDD two-tier HetNet composed of $L$ cells, as depicted in Fig. 1,

wherein the main cell, referred to as cell 1 or macrocell, is equipped with a central macro base station (MBS). The remaining cells, $2, \ldots, L$, are endowed with a single lower power micro BS (mBS). For each cell $j, j=1, \ldots, L$, we call $\mathrm{BS}_{j}$ its corresponding BS and assume that it has $M_{j}$ antennas and serves $K_{j}$ mono-antenna users $\left(\mathrm{UEs}_{j}\right)$. The set of UEs associated with the macro-cell, $\mathrm{UEs}_{1}$, are distributed uniformly over the whole coverage area of the network. As for $j=2, \ldots, L, \mathrm{UEs}_{j}$ are uniformly spread over the coverage area of cell $j$ which is delimited by a circle of radius $R$. We assume that all BSs use Gaussian codebooks and linear precoding. Based on this assumption, $\mathrm{BS}_{j}$ transmits the signal

$$
\mathbf{x}_{j}=\sum_{k=1}^{K_{j}} \mathbf{w}_{j, k} s_{j, k}
$$

where $s_{j, k} \sim \mathcal{C N}(0,1)$ is the downlink data symbol intended to UE $k$ in cell $j$ and $\mathbf{w}_{j, k}$ its associated precoding vector.

We denote by $\mathbf{h}_{\ell, j, k}$ the channel vector between $\mathrm{BS}_{\ell}$ and UE $j$ in cell $k$. The received signal by UE $j$ in cell $k$ can be expressed as:

$$
y_{j, k}=\sum_{\ell=1}^{L} \sum_{k=1}^{K_{\ell}} \mathbf{h}_{\ell, j, k}^{\mathrm{H}} \mathbf{w}_{\ell, i} s_{\ell, i}+\mathbf{n}_{j, k}
$$

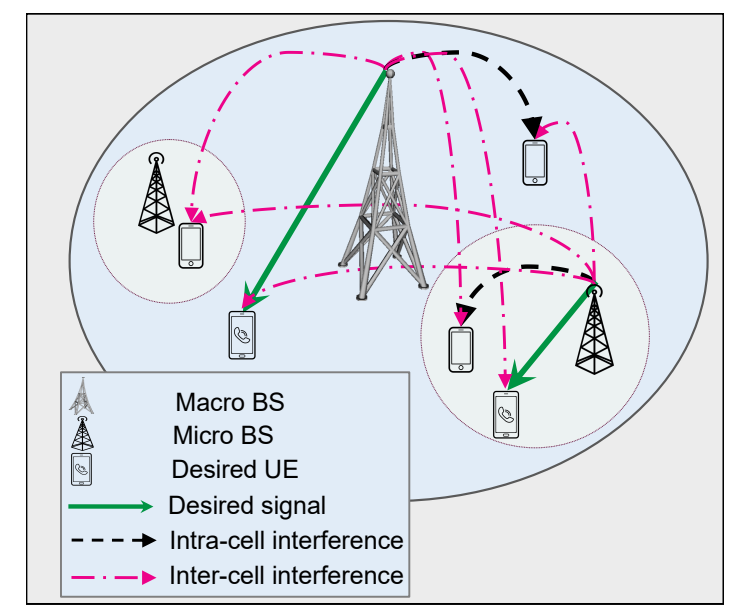

Fig. 1. Two-Tier HetNet with legitimate and different types of interfering channels.

where $\mathbf{n}_{j, k} \sim \mathcal{C N}\left(0, \sigma^{2} \mathbf{I}_{M_{j}}\right)$ accounts for the additive Gaussian noise. We can further decompose $y_{j, k}$ as:

$y_{j, k}=$

$\mathbf{h}_{j, j, k}^{\mathrm{H}} \mathbf{w}_{j, k} s_{j, k}+\sum_{\substack{i=1 \\ i \neq k}}^{K_{j}} \mathbf{h}_{j, j, k}^{\mathrm{H}} \mathbf{w}_{j, i} s_{j, i}+\sum_{\substack{\ell=1 \\ \ell \neq j}}^{L} \sum_{i=1}^{K_{\ell}} \mathbf{h}_{\ell, j, k}^{\mathrm{H}} \mathbf{w}_{\ell, i} s_{\ell, i}+\mathbf{n}_{j, k}$,

This decomposition distinguishes the desired signal term from the overall interference and noise. Hence, the SINR at UE $k$ in cell $j$ writes as:

$$
\begin{aligned}
\operatorname{SINR}_{j, k} & =\frac{\left|\mathbf{h}_{j, j, k}^{\mathrm{H}} \mathbf{w}_{j, k}\right|^{2}}{\sum_{\substack{i=1 \\
i \neq k}}^{K_{j}}\left|\mathbf{h}_{j, j, k}^{\mathrm{H}} \mathbf{w}_{j, i}\right|^{2}+\sum_{\substack{\ell=1 \\
\ell \neq j}}^{L} \sum_{i=1}^{K_{\ell}}\left|\mathbf{h}_{\ell, j, k}^{\mathrm{H}} \mathbf{w}_{\ell, i}\right|^{2}+\sigma^{2}} \\
& =\frac{S_{j, k}}{I_{j, k}^{\text {intra }}+\sum_{\ell=1}^{L} I_{\ell, j, k}^{\text {inter }}+\sigma^{2}}
\end{aligned}
$$

where

- $S_{j, k}=\left|\mathbf{h}_{j, j, k}^{\mathrm{H}} \mathbf{w}_{j, k}\right|^{2}$ is the received signal power at user $k$ in cell $j$,

- $I_{j, k}^{\text {intra }}=\sum_{i=1, i \neq k}^{K_{j}}\left|\mathbf{h}_{j, j, k}^{H} \mathbf{w}_{j, i}\right|^{2}$ is the received intra-cell interference at user $k$,

- $I_{\ell, j, k}=\sum_{i=1}^{K_{\ell}}\left|\mathbf{h}_{\ell, j, k}^{\mathrm{H}} \mathbf{w}_{\ell, i}\right|^{2}$ is the interference generated by cell $\ell$ to the user $k$ in cell $j$.

We are ultimately interested in the DL average achievable rate per UE in each cell $j, j=1, \cdots, L$, which is a commonly used performance metric based on the SINR values and straightforward to interpret. Under the assumption that interference is treated as noise, the DL average achievable rate per UE for cell $j$ is defined as:

$$
R_{j}=\frac{1}{K_{j}} \sum_{k=1}^{K_{j}} \mathbb{E}\left[\log _{2}\left(1+\operatorname{SINR}_{j, k}\right)\right]
$$


where the expectation is taken over the channels $\mathbf{h}_{j, \ell, k}$.

\section{B. Channel Model}

In this work, we consider the case in which the channels $\left\{\mathbf{h}_{j, \ell, k}\right\}$ are spatially uncorrelated, obeying the model:

$$
\mathbf{h}_{j, \ell, k}=\sqrt{P_{j, \ell, k}} \mathbf{z}_{j, \ell, k},
$$

where $\mathbf{z}_{j, \ell, k} \in \mathbb{C}^{M_{j} \times 1}$ is the complex Gaussian vector with i.i.d entries of zero mean and unit variance which accounts for the small-scale fading and $P_{j, \ell, k}$ refers to the amount of power received at $\mathrm{UE}_{j, k}$ from $\mathrm{BS}_{j}$. For each $\mathrm{BS}_{j}$, we assume that the available power $P_{j}$ is equally allocated among its legitimate UEs. In addition, for each UE $k$ in cell $j$, the received power undergoes some channel attenuation which we denote by $\alpha_{j, \ell, k}$. This implies that $P_{j, \ell, k}$ is given by:

$$
P_{j, \ell, k}=\frac{P_{j}}{K_{j}} \alpha_{j, \ell, k} .
$$

In practice, only an imperfect estimate $\widehat{\mathbf{h}}_{j, \ell, k}$ of the true channel $\mathbf{h}_{j, \ell, k}$ is available at the transmitter. This imperfection arises naturally from users mobility for instance. In the present work, we model imperfect CSI as follows :

$$
\widehat{\mathbf{h}}_{j, \ell, k}=\sqrt{P_{j, \ell, k}}\left(\sqrt{1-\tau_{j, \ell, k}^{2}} \mathbf{z}_{j, \ell, k}+\tau_{j, \ell, k} \mathbf{q}_{j, \ell, k}\right),
$$

where $\sqrt{P_{j, \ell, k}} \mathbf{q}_{j, \ell, k} \sim \mathcal{C N}\left(0, P_{j, \ell, k} \mathbf{I}_{M_{j}}\right)$ accounts for the channel estimation errors and is independent of $\mathbf{h}_{j, \ell, k}$. The parameter $\tau_{j, \ell, k} \in[0,1]$ reflects the accuracy of the channel estimate such that $\tau_{j, \ell, k}=0$ refers to perfect CSI, whereas for $\tau_{j, \ell, k}=1$, the estimate is completely independent of the real channel.

We denote the aggregate channel matrix linking $\mathrm{BS}_{j}$ to users in cell $\ell$ by $\mathbf{H}_{j, \ell}=\left[\mathbf{h}_{j, \ell, 1}, \mathbf{h}_{j, \ell, 2}, \ldots, \mathbf{h}_{j, \ell, K_{\ell}}\right]$. Accordingly, the aggregate channel estimates matrix is given by :

$$
\widehat{\mathbf{H}}_{j, \ell}=\left[\widehat{\mathbf{h}}_{j, \ell, 1}, \widehat{\mathbf{h}}_{j, \ell, 2}, \ldots, \widehat{\mathbf{h}}_{j, \ell, K_{\ell}}\right]
$$

\section{Coordinated SLNR-MAX Design}

In a multi-cell system, inter-cell interference caused by the transmissions of neighboring cells, can seriously affect the downlink performances, especially that of users at the cell boundaries. To combat inter-cell interference, one possible way consists in using coordinated beamforming designs that attempt to mitigate the interference caused by each BS to non-intended users. Several levels of coordination can be envisioned. In this paper, we consider the case in which each BS sends data to its own UEs, and estimates its channels with its UEs and UEs in other cells. If perfect CSI is available, complete elimination of both intra-cell and inter-cell interference can be accomplished by coordinated zero-forcing beamforming (ZFBF). However, this requires the $\mathrm{BS}$ to possess a sufficient number of antennas, and more importantly, is not necessarily optimal, as it may entail a considerable loss in the energy of the desired signal.To create a good balance between interference and signal, other coordinated precoding techniques should be used [18]. Of interest in this paper is the class of precoding maximizing the signal to leakage and noise ratio, which we denote by
SLNR-MAX. This class can be also used when coordination is not employed. In this case, the signal to leakage ratio is obtained by considering only the interference leakage to the other UEs in the same cell, thus yielding:

$$
\mathrm{SLNR}_{j, k}^{\mathrm{no}-\mathrm{co}}=\frac{\left|\mathbf{h}_{j, j, k}^{\mathrm{H}} \mathbf{w}_{j, k}\right|^{2}}{\sum_{\substack{i=1 \\ i \neq k}}^{K_{j}}\left|\mathbf{h}_{j, j, i}^{\mathrm{H}} \mathbf{w}_{j, k}\right|^{2}+\rho \sigma^{2}},
$$

where $\rho$ herein is a regularization parameter weighting the noise energy. It can be easily shown that the precoding vector maximizing (10) is given by:

$$
\mathbf{w}_{j, k}^{\text {no-co }}=\frac{\left(\mathbf{H}_{j, j} \mathbf{H}_{j, j}^{\mathrm{H}}+\rho \sigma^{2} \mathbf{I}_{M_{j}}\right)^{-1} \mathbf{h}_{j, j, k}}{\left\|\left(\mathbf{H}_{j, j} \mathbf{H}_{j, j}^{\mathrm{H}}+\rho \sigma^{2} \mathbf{I}_{M_{j}}\right)^{-1} \mathbf{h}_{j, j, k}\right\|},
$$

In the coordinated case, the total amount of interference leakage caused by the serving BS is taken into account. In particular, the SLNR incurred by the communication between cell $j$ and its $\mathrm{UE} k$ is given by:

$$
\begin{aligned}
& \operatorname{SLNR}_{j, k}^{\mathrm{co}}= \\
& \frac{\left|\mathbf{h}_{j, j, k}^{\mathrm{H}} \mathbf{w}_{j, k}\right|^{2}}{\sum_{\substack{i=1 \\
i \neq k}}^{K_{j}}\left|\mathbf{h}_{j, j, i}^{\mathrm{H}} \mathbf{w}_{j, k}\right|^{2}+\sum_{\substack{\ell=1 \\
\ell \neq j}}^{L} \sum_{i=1}^{K_{\ell}}\left|\mathbf{h}_{j, \ell, i}^{\mathrm{H}} \mathbf{w}_{j, k}\right|^{2}+\rho \sigma^{2}}, \\
& =\frac{S_{j, k}}{\mathcal{L}_{j, k}^{\text {intra }}+\sum_{\ell=1, \ell \neq j}^{L} \mathcal{L}_{j, k}^{\text {inter }, \ell}+\rho \sigma^{2}}
\end{aligned}
$$

where:

- $\mathcal{L}_{j, k}^{\text {intra }}$ is the interference leakage generated by the communication between $\mathrm{BS}_{j}$ and UE $k$ to all UEs $i \neq k$ in

- ${ }^{\mathcal{L}_{j, k}^{\text {inter }, \ell},}$ is the interference leakage caused by the communication between $\mathrm{BS}_{j}$ and its UE $k$ to all UEs in cell $\ell$.

It can be easily proven that the beamforming vector maximizing (13) is given by:

$$
\mathbf{w}_{j, k}^{\mathrm{co}}=\frac{\mathbf{v}_{j, k}^{\mathrm{co}}}{\left\|\mathbf{v}_{j, k}^{\mathrm{co}}\right\|}, \quad \text { s.t. }
$$

$$
\mathbf{v}_{j, k}^{\text {co }}=\left(\mathbf{H}_{j, j} \mathbf{H}_{j, j}^{\mathrm{H}}+\sum_{\substack{\ell=1 \\ \ell \neq j}} \mathbf{H}_{j, \ell} \mathbf{H}_{j, \ell}^{\mathrm{H}}+\rho \sigma^{2} \mathbf{I}_{M_{j}}\right)^{-1} \mathbf{h}_{j, j, k} .
$$

Proof: Consider the problem :

$$
\mathbf{w}_{j, k}^{\mathrm{co}}=\underset{\mathbf{w}_{j, k}}{\operatorname{argmax}} \quad \mathrm{SLNR}_{j, k}^{\mathrm{co}} \quad \text { subject to }\left|\mathbf{w}_{j, k}\right|^{2}=1 .
$$


Which can be rewritten as:

$$
\underset{\mathbf{w}_{j, k}^{\text {co }}}{\underset{j, k}{\operatorname{argmax}}} \frac{\mathbf{w}_{j, k}^{\mathrm{H}} \mathbf{h}_{j, j, k} \mathbf{h}_{j, j, k}^{\mathrm{H}} \mathbf{w}_{j, k}}{\mathbf{w}_{j, k}^{\mathrm{H}}\left(\mathbf{H}_{j, j} \mathbf{H}_{j, j}^{\mathrm{H}}+\sum_{\substack{\ell=1 \\ \ell \neq j}}^{L} \mathbf{H}_{j, \ell} \mathbf{H}_{j, \ell}^{\mathrm{H}}+\rho \sigma^{2}\right)^{-1} \mathbf{w}_{j, k}},
$$

subject to $\left|\mathbf{w}_{j, k}\right|^{2}=1$. The expression in (16) is a ratio of two quadratic forms. Therefore, the solution to $(\mathrm{P})$ is given by:

$\mathbf{w}_{j, k}^{c o} \propto$

max eig.vect. $\left[\left(\mathbf{H}_{j, j} \mathbf{H}_{j, j}^{\mathrm{H}}+\sum_{\substack{\ell=1 \\ \ell \neq j}}^{L} \mathbf{H}_{j, \ell} \mathbf{H}_{j, \ell}^{\mathrm{H}}+\rho \sigma^{2}\right)^{-1} \mathbf{h}_{j, j, k} \mathbf{h}_{j, j, k}^{\mathrm{H}}\right]$.

Since $\operatorname{rank}\left(\mathbf{h}_{j, j, k} \mathbf{h}_{j, j, k}^{\mathrm{H}}\right)=1$, we find that ${ }^{1}$ :

$$
\mathbf{w}_{j, k}^{c o} \propto\left(\mathbf{H}_{j, j} \mathbf{H}_{j, j}^{\mathrm{H}}+\sum_{\substack{\ell=1 \\ \ell \neq j}}^{L} \mathbf{H}_{j, \ell} \mathbf{H}_{j, \ell}^{\mathrm{H}}+\rho \sigma^{2}\right)^{-1} \mathbf{h}_{j, j, k}
$$

Finally, to satisfy the constraint of unitary norm, $\mathbf{w}_{j, k}^{c o}$ is chosen as in (14).

If only imperfect CSI is available at the BSs, we propose to simply replacing the true channels $\mathbf{h}_{\ell, j, k}$ by their estimates in the expressions of $\mathbf{w}_{j, k}^{\mathrm{no}-\mathrm{co}}$ and $\mathbf{w}_{j, k}^{\mathrm{co}}$. In doing so, the beamforming vector $\mathbf{w}_{j, k}^{\text {no }}$-co takes the form:

$$
\widehat{\mathbf{w}}_{j, k}^{\text {no-co }}=\frac{\widehat{\mathbf{v}}_{j, k}^{\text {no-co }}}{\left\|\widehat{\mathbf{v}}_{j, k}^{\text {no-co }}\right\|}, \quad \text { s.t. },
$$

with $\widehat{\mathbf{v}}_{j, k}^{\text {no-co }}=\left(\widehat{\mathbf{H}}_{j, j} \widehat{\mathbf{H}}_{j, j}^{\mathrm{H}}+\rho \sigma^{2} \mathbf{I}_{M_{j}}\right)^{-1} \widehat{\mathbf{h}}_{j, j, k}$. In the coordinated case, we pursue the same approach with the single difference that the channels with UEs in other cells are weighted by the coordination factor $\beta_{j}$.

$$
\widehat{\mathbf{w}}_{j, k}^{\mathrm{co}}=\frac{\widehat{\mathbf{v}}_{j, k}^{\mathrm{co}}}{\left\|\widehat{\mathbf{v}}_{j, k}^{\mathrm{co}}\right\|}, \quad \text { s.t. }
$$

with

$$
\widehat{\mathbf{v}}_{j, k}^{\mathrm{co}}=\left(\widehat{\mathbf{H}}_{j, j} \widehat{\mathbf{H}}_{j, j}^{\mathrm{H}}+\beta_{j} \sum_{\substack{\ell=1 \\ \ell \neq j}} \widehat{\mathbf{H}}_{j, \ell} \widehat{\mathbf{H}}_{j, \ell}^{\mathrm{H}}+\rho \sigma^{2} \mathbf{I}_{M_{j}}\right)^{-1} \mathbf{h}_{j, j, k} .
$$

The reason behind using the factor $\beta_{j}$ is that channel estimates with UEs in other cells might be unreliable. The coordination factor $\beta_{j}$ is thus used to place more or less importance on these estimates.

\footnotetext{
${ }^{1} \operatorname{rank}(A B) \leq \min \{\operatorname{rank}(A), \operatorname{rank}(B)\}$
}

Another advantage of studying the performances of SLNRMAX beamforming is that it encompasses other types of precoding designs by properly selecting the parameters $\beta_{j}$ and $\rho$. For instance, ZFBF can be obtained by setting $\beta_{j}=1$ and taking the limit of (11) and (21) as $\rho \rightarrow 0$.

\section{ASYMPTOTIC SINR AND SLNR ANALYSIS}

This section presents an asymptotic performance analysis of the expressions of the SINR and SLNR. Our results are valid in the asymptotic regime in which the number of antennas $M_{i}$ and that of users $K_{i}$ grow large at the same pace. More formally, this assumption can be written as:

Assumption 1. We assume that $L$ is fixed. Define $c_{i}=\frac{K_{i}}{M_{i}}$. Then, we have:

$$
0<\liminf c_{i}<\lim \sup c_{i}<\infty
$$

Furthermore, we shall additionally assume that for all $j, \ell, k$, $P_{j} \alpha_{j, \ell, k}$ remains bounded. This can be formulated as:

Assumption 2. $\forall j, \ell$ and $k$,

$$
0<\liminf \min _{j, \ell, k} P_{j} \alpha_{j, \ell, k} \leq \lim \sup \max _{j, \ell, k} P_{j} \alpha_{j, \ell, k}<\infty
$$

Let $K=\sum_{i=1}^{L} K_{i}$. For simplicity, the asymptotic regime in Assumption 1, will be denoted by $K \rightarrow \infty$.

\section{A. Deterministic Equivalents for the SINR}

In this section, we derive closed-form approximations for the SINR. Our main results are stated in two theorems, treating separately the cases in which the underlying BS coordinates or not. In both theorems, we will provide for each cell $j$, accurate approximations of the signal power $S_{j, k}$ and the intra-cell interference $I_{j, k}^{\text {intra }}$ received by its associated user $k$ as well as the inter-cell interference $\left\{I_{j, \ell, k}^{\text {inter }}, k=1, \cdots, K_{\ell}\right\}$ that it generates to every cell $\ell, \ell \neq j$. We find it the most informative to analyze separately each term rather than the overall expression of the SINR. As will be explained later, gathering these results will easily lead to the analysis of any possible network configuration. Plus, using the continuous mapping theorem in [28], the deterministic equivalent of the SINR is obtained by the ratio between the respective approximations of the signal power and total interference plus noise derived in Theorems 1 and 2.

We will consider first the case in which $\mathrm{BS}_{j}$ does not perform coordination. To disntguish this case, we will use the superscript no-co in all our derivations.

Prior to presenting our main results, we shall introduce deterministic equivalents that are commonly used within the framework of random matrix theory. These quantities will be needed to express deterministic approximations of the SINR and the SLNR. 
For $t>0$ and $j \in\{1, \cdots, L\}$, we define $e_{j}^{\mathrm{no}-\mathrm{co}}(t)$ as the unique positive solution to the following fixed-point equation:

$$
e_{j}^{\mathrm{no}-\mathrm{co}}(t)=\frac{P_{j}}{K_{j}} \sum_{i=1}^{K_{j}} \frac{\alpha_{j, j, i}}{t+\frac{1}{c_{j}} P_{j} \alpha_{j, j, i} \tilde{e}_{j}^{\mathrm{no}-\mathrm{co}}(t)},
$$

with,

$$
\tilde{e}_{j}^{\mathrm{no}-\mathrm{co}}(t)=\frac{1}{1+e_{j}^{\mathrm{no}-\mathrm{co}}(t)} .
$$

The existence and uniqueness of $e_{j}^{\text {no-co }}$ follows from standard results of random matrix theory (See Proposition 1 in Appendix A.) Furthermore, we will need the following deterministic quantities:

$$
\Gamma_{j, \ell, i}^{\mathrm{no}-\mathrm{co}}\left(t, c_{j}\right)=\frac{1}{1+\frac{t c_{j}}{P_{j} \alpha_{j, \ell, i} \tilde{e}_{j}^{\mathrm{no}-\mathrm{co}}(t)}},
$$

and:

$$
\tilde{\vartheta}_{j}^{\mathrm{no}-\mathrm{co}}(t)=\frac{c_{j}^{2}}{K_{j}} \sum_{i=1}^{K_{j}}\left[\Gamma_{j, j, i}^{\mathrm{no}-\mathrm{co}}\left(t, c_{j}\right)\right]^{2} .
$$

We are now ready to state our first main result.

Theorem 1. Let Assumptions 1 and 2 hold. Define for $t>0$ and $j \in\{1, \cdots, L\}, e_{j}^{\mathrm{no}-\mathrm{co}}(t)$ as in (22) and consider the notations (23)-(24). Let $\bar{S}_{j, k}^{\mathrm{no}-\mathrm{co}}, \bar{I}_{j, k}^{\mathrm{intra}, \mathrm{no}-\mathrm{co}}$ and $\bar{I}_{j, \ell, k}^{\mathrm{inter}, \mathrm{no}-\mathrm{co}}$ be given by

$\bar{S}_{j, k}^{\mathrm{no}-\mathrm{co}}=P_{j} \alpha_{j, j, k} \frac{\left(1-\tau_{j, j, k}^{2}\right)}{c_{j}}\left[1-\frac{1}{c_{j}} \tilde{\vartheta}_{j}^{\mathrm{no}-\mathrm{co}}\left(\rho \sigma^{2}\right)\right]$,

$\bar{I}_{j, k}^{\mathrm{intra}, \mathrm{no}-\mathrm{co}}=P_{j} \alpha_{j, j, k}\left[\left(1-\tau_{j, j, k}^{2}\right)\left(1-\Gamma_{j, j, k}^{\mathrm{no}-\mathrm{co}}\left(\rho \sigma^{2}, c_{j}\right)\right)^{2}+\tau_{j, j, k}^{2}\right]$

$\bar{I}_{j, \ell, k}^{\mathrm{inter}, \mathrm{no}-\mathrm{co}}=P_{j} \alpha_{j, \ell, k}$.

Then,

$$
\begin{aligned}
& \max _{1 \leq k \leq K_{j}}\left|S_{j, k}^{\text {no-co }}-\bar{S}_{j, k}^{\text {no-co }}\right| \underset{K \rightarrow \infty}{\stackrel{a . s .}{\longrightarrow}} 0, \\
& \max _{1 \leq k \leq K_{j}}\left|I_{j, k}^{\text {intra,no-co }}-\bar{I}_{j, k}^{\text {intra,no-co }}\right| \underset{K \rightarrow \infty}{\stackrel{a . s .}{\longrightarrow}} 0, \\
& \max _{1 \leq k \leq K_{j}}\left|I_{j, \ell, k}^{\text {inter,no-co }}-\bar{I}_{j, \ell, k}^{\text {inter,no-co }}\right| \underset{K \rightarrow \infty}{\stackrel{a . s .}{\longrightarrow}} 0 .
\end{aligned}
$$

Proof: The proof of Theorem 1 is given in Appendix B.

Corollary 1. If perfect CSI of channel $\mathbf{h}_{j, j, k}$ is available, i.e. for $\tau_{j, j, k}=0$, the asymptotic expressions $\bar{S}_{j, k}^{\mathrm{no}-\mathrm{co}}, \bar{I}_{j, k}^{\mathrm{intr}}$ and $\bar{I}_{j, \ell, k}^{\text {inter,no-co }}$ become :

$$
\begin{aligned}
\bar{S}_{j, k}^{\mathrm{no}-\mathrm{co}} & =\frac{P_{j} \alpha_{j, j, k}}{c_{j}}\left(1-\frac{1}{c_{j}} \tilde{\vartheta}_{j}^{\mathrm{no}-\mathrm{co}}\left(\rho \sigma^{2}\right)\right), \\
\bar{I}_{j, k}^{\text {intra,no-co }} & =\frac{P_{j} \alpha_{j, j, k}}{\left(1+\frac{1}{\rho \sigma^{2} c_{j}} P_{j} \alpha_{j, j, k} \tilde{e}_{j}^{\mathrm{no}-\mathrm{co}}\left(\rho \sigma^{2}\right)\right)^{2}}, \\
\bar{I}_{j, \ell, k}^{\text {inter,no-co }} & =P_{j} \alpha_{j, \ell, k} .
\end{aligned}
$$

Some important insights can be readily extracted from Theorem 1 and corollary 1 . First, we note that $\bar{S}_{j, k}^{\text {no-co }}$,
$\bar{I}_{j, k}^{\text {intra,no-co }}$ and $\bar{I}_{j, \ell, k}^{\text {inter,no-co }}$ depend only on the large-scale channel fading parameters, and thus are instructive in how these parameters affect performances. In particular, we can easily obtain the asymptotic quantities when ZFBF is used by simply taking the limit of the provided expressions as $\rho$ tends to zero. In doing so, we obtain the following expressions ${ }^{2}$ :

$$
\begin{aligned}
& {\left[\bar{S}_{j, k}^{\mathrm{no}-\mathrm{co}}\right]_{\mathrm{ZF}}=\frac{\left(1-\tau_{j, j, k}^{2}\right)}{c_{j}} P_{j} \alpha_{j, j, k}\left(1-c_{j}\right),} \\
& {\left[\bar{I}_{j, k}^{\text {intra,no-co }}\right]_{\mathrm{ZF}}=\tau_{j, j, k}^{2} P_{j} \alpha_{j, j, k} .} \\
& {\left[\bar{I}_{j, \ell, k}^{\text {inter,no-co }}\right]_{\mathrm{ZF}}=P_{j} \alpha_{j, \ell, k} .}
\end{aligned}
$$

Comparing (26) with (32) and (27) with (33), we can easily see that:

$$
\begin{aligned}
& {\left[\bar{S}_{j, k}^{\mathrm{no}-\mathrm{co}}\right]_{\mathrm{SLNR}-\mathrm{MAX}}>\left[\bar{S}_{j, k}^{\mathrm{no}-\mathrm{co}}\right]_{\mathrm{ZF}},} \\
& {\left[\bar{I}_{j, k}^{\mathrm{intra}, \mathrm{no}-\mathrm{co}}\right]_{\mathrm{SLNR}-\mathrm{MAX}}>\left[\bar{I}_{j, k}^{\mathrm{intra}, \mathrm{no}-\mathrm{co}}\right]_{\mathrm{ZF}},}
\end{aligned}
$$

This proves that ZFBF completely cancels the intra-cell interference in the perfect CSI case $\left(\tau_{j, j, k}=0\right)$, but induces a loss in the signal power as compared to the SLNR-MAX.

We will now consider the case in which $\mathrm{BS}_{j}$ performs coordination. Similarly, we use the superscript co to distinguish this case and introduce the following deterministic quantities that will serve later to express closed-form expressions of the SINR:

Let $e_{j}^{\mathrm{co}}(t)$ be the unique solution to the following fixed point ,equation:

$e_{j}^{\mathrm{co}}(t)=$
$\frac{P_{j}}{K}\left[\sum_{i=1}^{K_{j}} \frac{\alpha_{j, j, i}}{t+\frac{1}{\tilde{c}_{j}} P_{j} \alpha_{j, j, i} \tilde{e}_{j}^{\mathrm{co}}(t)}+\sum_{\substack{\ell=1 \\ \ell \neq j}}^{L} \sum_{i=1}^{K_{\ell}} \frac{\beta_{j} \alpha_{j, \ell, i}}{t+\frac{\beta_{j}}{\tilde{c}_{j}} P_{j} \alpha_{j, \ell, i} \tilde{e}_{j}^{\mathrm{co}}(t)}\right]$,

with $\tilde{c}_{j} \triangleq \frac{K}{M_{j}}$ and $\tilde{e}_{j}^{\mathrm{co}}(t)=\left(1+e_{j}^{\mathrm{co}}(t)\right)^{-1}$. Let

$$
t_{\mathrm{co}}=\frac{K_{j}}{K} \rho \sigma^{2} \text {. }
$$

Define also $\Gamma_{j, \ell, i}^{\mathrm{co}}\left(t, c_{j}\right), \Psi_{j, \ell, i}^{\mathrm{co}}\left(t, c_{j}\right)$ and $\tilde{\vartheta}_{j}^{\mathrm{co}}(t)$ as:

$$
\begin{aligned}
& \Gamma_{j, \ell, i}^{\mathrm{co}}\left(t, c_{j}\right)=\frac{1}{1+\frac{t c_{j}}{P_{j} \alpha_{j, \ell, i} \tilde{e}_{j}^{\mathrm{co}}(t)}} \\
& \Psi_{j, \ell, i}^{\mathrm{co}}\left(t, c_{j}\right)=\frac{1}{1+\frac{t c_{j}}{\beta_{j} P_{j} \alpha_{j, \ell, i} \tilde{e}_{j}^{\mathrm{co}}(t)}}, \\
& \tilde{\vartheta}_{j}^{\mathrm{co}}(t)=\frac{\tilde{c}_{j}^{2}}{K}\left[\sum_{i=1}^{K_{j}}\left(\Gamma_{j, j, i}^{\mathrm{co}}\left(t, \tilde{c}_{j}\right)\right)^{2}+\sum_{\substack{\ell=1 \\
\ell \neq j}}^{L} \sum_{i=1}^{K_{\ell}}\left(\Psi_{j, \ell, i}^{\mathrm{co}}\left(t, \tilde{c}_{j}\right)\right)^{2}\right] .
\end{aligned}
$$

With these notations at hand, we are now ready to state our second main result, which provides deterministic equivalents

\footnotetext{
${ }^{2}$ This can be easily shown by noting that as $t$ tends to zero, $\Gamma_{j, \ell, i}^{\mathrm{no}-\mathrm{co}}(t) \rightarrow 1$
} and $\tilde{\vartheta}_{j}^{\mathrm{no}-\mathrm{co}}(t) \rightarrow c_{j}^{2}$. 
for $S_{j, k}^{\mathrm{co}}, I_{j, k}^{\mathrm{intra}, \mathrm{co}}$ and $I_{j, \ell, k}^{\mathrm{inter}, \mathrm{co}}$.

Theorem 2. Let Assumptions 1 and 2 hold true. Let $\bar{S}_{j, k}^{\mathrm{co}}$, $\bar{I}_{j, k}^{\text {intra,co }}$ and $\bar{I}_{j, \ell, k}^{\text {inter,co }}$ be given by

$\bar{S}_{j, k}^{\mathrm{co}} \quad=P_{j} \alpha_{j, j, k} \frac{\left(1-\tau_{j, j, k}^{2}\right)}{c_{j}}\left[1-\frac{1}{\tilde{c}_{j}} \tilde{\vartheta}_{j}^{\mathrm{co}}\left(t_{\mathrm{co}}\right)\right]$,

$\bar{I}_{j, k}^{\mathrm{intra}, \mathrm{co}}=P_{j} \alpha_{j, j, k}\left[\left(1-\tau_{j, j, k}^{2}\right)\left(1-\Gamma_{j, j, k}^{\mathrm{co}}\left(t_{\mathrm{co}}, \tilde{c}_{j}\right)\right)^{2}+\tau_{j, j, k}^{2}\right]$,

$\bar{I}_{j, \ell, k}^{\text {inter co }}=P_{j} \alpha_{j, \ell, k}\left[\left(1-\tau_{j, \ell, k}^{2}\right)\left(1-\Psi_{j, \ell, k}^{\mathrm{co}}\left(t_{\mathrm{co}}, \tilde{c}_{j}\right)\right)^{2}+\tau_{j, \ell, k}^{2}\right]$

Then,

$$
\begin{aligned}
& \max _{1 \leq k \leq K_{j}}\left|S_{j, k}^{\mathrm{co}}-\bar{S}_{j, k}^{\mathrm{co}}\right| \stackrel{\text { a.s. }}{\underset{K \rightarrow \infty}{\longrightarrow}} 0, \\
& \max _{1 \leq k \leq K_{j}}\left|I_{j, k}^{\text {intra,co }}-\bar{I}_{j, k}^{\text {intra,co }}\right| \underset{\text { a.s. }}{\stackrel{\text { In }}{\longrightarrow} 0} 0, \\
& \max _{1 \leq k \leq K_{j}}\left|I_{j, \ell, k}^{\text {inter,co }}-\bar{I}_{j, \ell, k}^{\text {inter,co }}\right| \underset{K \rightarrow \infty}{\stackrel{\text { a.s. }}{\longrightarrow} 0 .}
\end{aligned}
$$

Proof: The proof of Theorem 2 is given in Appendix $\mathrm{B}[$ part II].

Corollary 2. When the channel is perfectly known at all BSs, $\left(\tau_{j, \ell, k}=0 \forall j, \ell, k\right)$, we have then :

$$
\begin{aligned}
& \bar{S}_{j, k}^{\mathrm{co}}=\frac{P_{j} \alpha_{j, j, k}}{c_{j}}\left(1-\frac{1}{\tilde{c}_{j}} \tilde{\vartheta}_{j}^{\mathrm{co}}\right), \\
& \bar{I}_{j, k}^{\mathrm{intra}, \mathrm{co}}=\frac{P_{j} \alpha_{j, j, k}}{\left(1+\frac{1}{c_{j} \rho \sigma^{2}} P_{j} \alpha_{j, j, k} \tilde{e}_{j}^{\mathrm{co}}\left(t_{\mathrm{co}}\right)\right)^{2}}, \\
& \bar{I}_{j, \ell, k}^{\text {inter }, \mathrm{co}}=\frac{P_{j} \alpha_{j, \ell, k}}{\left(1+\frac{\beta_{j}}{c_{j} \rho \sigma^{2}} P_{j} \alpha_{j, \ell, k} \tilde{e}_{j}^{\mathrm{co}}\left(t_{\mathrm{co}}\right)\right)^{2}} .
\end{aligned}
$$

Similar to above, we stress the fact that the closed-form approximations of the signal and intereference terms provided by Theorem 2 depend solely on the large-scale fading parameters. Furthermore, our results can be used to analyze the case in which $\mathrm{BS}_{j}$ employs ZFBF by taking the limit as $\rho$ tends to zero. In doing so, we get:

$$
\begin{aligned}
& {\left[\bar{S}_{j, k}^{\mathrm{co}}\right]_{\mathrm{ZF}}=\frac{\left(1-\tau_{j, j, k}^{2}\right)}{c_{j}} P_{j} \alpha_{j, j, k}\left(1-\tilde{c}_{j}\right),} \\
& {\left[\bar{I}_{j, k}^{\text {intra,co }}\right]_{\mathrm{ZF}}=\tau_{j, j, k}^{2} P_{j} \alpha_{j, j, k},} \\
& {\left[\bar{I}_{j, \ell, k}^{\text {inter }, \mathrm{co}}\right]_{\mathrm{ZF}}=\tau_{j, \ell, k}^{2} P_{j} \alpha_{j, \ell, k} .}
\end{aligned}
$$

Theorem 2 sheds light on the impact of $\mathrm{BS}_{j}$ performing coordination on the reduction of the inter-cell interference term $\bar{I}_{j, \ell, k}^{\text {inter,co }}$

Indeed, it follows from some mathematical manipulations that $^{3} \bar{I}_{j, \ell, k}^{\text {inter,co }}<\bar{I}_{j, \ell, k}^{\text {inter,no-co }}$.

Also, from (40), (44) and (47), we can clearly see that taking $\beta_{j}$ large would lead to the vanishing of the inter-cell interference. This however may be not necessarily optimal,

\footnotetext{
${ }^{3}$ It is shown by noting that:
for $0<\tau_{j, \ell, k}<1,\left(1-\tau_{j, \ell, k}^{2}\right)\left(1-\Psi_{j, \ell, k}^{\mathrm{co}}\left(t_{\mathrm{co}}, \tilde{c}_{j}\right)\right)^{2}+\tau_{j, \ell, k}^{2}<1$
}

since it might cause a considerable loss in the signal power. On another note, it seems that there is little interest to perform coordination when the channel estimation quality is poor. This can be clearly understood by observing that as $\tau_{j, \ell, k} \rightarrow 1$, $\bar{I}_{j, \ell, k}^{\text {inter,co }} \rightarrow \bar{I}_{j, \ell, k}^{\text {inter,no-co }}$. It is thus of major importance to "regulate" the coordination level in the precoding based on the quality of the available CSI. This will be the objective of the next section.

Lastly, we shall point out that the expressions in Theorems 1 and 2 can be used to analyse any possible coordination configuration. Let $\mathcal{S}_{c o}$ be the set containing the indexes of BSs that perform coordination, and $\mathcal{S}_{\text {no-co }}$ be that of BSs that do not employ coordination. Then, the asymptotic approximations of the SINRs for users associated with cells in $\mathcal{S}_{c o}$ and $\mathcal{S}_{n o-c o}$ are given by:

- User $k$ in $\mathrm{BS}_{j}$ where $j \in \mathcal{S}_{n o-c o}$ :

$\overline{\operatorname{SINR}}_{j, k}=$

$\frac{\bar{S}_{j, k}^{\text {no-co }}}{\bar{I}_{j, k}^{\text {intra,no-co }}+\sum_{\ell \in \mathcal{S}_{n o-c o}-\{j\}} \bar{I}_{\ell, j, k}^{\text {inter,no-co }}+\sum_{\ell \in \mathcal{S}_{c o}} \bar{I}_{\ell, j, k}^{\text {inter,co }}+\sigma^{2}}$.

- User $k$ in $\mathrm{BS}_{j}$ where $j \in \mathcal{S}_{c o}$ :

$$
\begin{aligned}
& \overline{\operatorname{SINR}}_{j, k}= \\
& \frac{\bar{S}_{j, k}^{\mathrm{co}}}{\bar{I}_{j, k}^{\text {intra,co }}+\sum_{\ell \in \mathcal{S}_{n o-c o}} \bar{I}_{\ell, j, k}^{\text {inter,no-co }}+\sum_{\ell \in \mathcal{S}_{c o}-\{j\}} \bar{I}_{\ell, j, k}^{\text {inter,co }}+\sigma^{2}} .
\end{aligned}
$$

The heterogeneity of the network can reside in which tier adopts coordinated beamforming and which one performs single-cell processing. This statement is analytically evidenced in equations (51) and (52) where the non-coordinated and coordinated precoding designs are combined in order to establish different strategies of coordination among tiers.

Case study: Since the macro BS is usually the main source of interference, a natural configuration would be when only the macro uses coordinated $\mathrm{BF}$ in order to minimize the interference it generates to the microcells users. In this case $\mathcal{S}_{c o}=\{1\}$ and $\mathcal{S}_{\text {no-co }}=\{2, \ldots, L\}$.

\section{B. Deterministic Equivalents for the SLNR}

In this section, we derive closed-form approximations of the $\left\{\mathrm{SLNR}_{j, k}\right\}$ in (13). As will be shown next, this result can be leveraged to determine a proper way of selecting the regularization factor $\beta_{j}$.

Theorem 3. Define, using the same notations as in Theorem 


\section{2, the following quantities:}

$\bar{S}_{j, k}^{\mathrm{co}}=P_{j} \alpha_{j, j, k} \frac{\left(1-\tau_{j, j, k}^{2}\right)}{c_{j}}\left[1-\frac{1}{\tilde{c}_{j}} \tilde{\vartheta}_{j}^{\mathrm{co}}\left(t_{\mathrm{co}}\right)\right]$,

$\overline{\mathcal{L}}_{j, k}^{\text {intra }}=\sum_{\substack{i=1 \\ i \neq k}}^{K_{j}} \frac{P_{j} \alpha_{j, j, i}}{K_{j}}\left[\left(1-\tau_{j, j, i}^{2}\right)\left(1-\Gamma_{j, j, i}^{\mathrm{co}}\left(t_{\mathrm{co}}, \tilde{c}_{j}\right)\right)^{2}+\tau_{j, j, i}^{2}\right]$,

$\overline{\mathcal{L}}_{j, k}^{\text {inter }, \ell}=\sum_{i=1}^{K_{\ell}} \frac{P_{j} \alpha_{j, \ell, i}}{K_{j}}\left[\left(1-\tau_{j, \ell, i}^{2}\right)\left(1-\Psi_{j, \ell, i}^{\mathrm{co}}\left(t_{\mathrm{co}}, \tilde{c}_{j}\right)\right)^{2}+\tau_{j, \ell, i}^{2}\right]$

Then, we have

$$
\begin{aligned}
& \max _{1 \leq k \leq K_{j}}\left|S_{j, k}^{\mathrm{co}}-\bar{S}_{j, k}^{\mathrm{co}}\right| \quad \underset{K \rightarrow \infty}{\stackrel{a . s .}{\longrightarrow}} 0 \\
& \max _{1 \leq k \leq K_{j}}\left|\mathcal{L}_{j, k}^{\text {intra }}-\overline{\mathcal{L}}_{j, k}^{\text {intra }}\right| \underset{K \rightarrow \infty}{\stackrel{\text { a.s. }}{\longrightarrow}} 0, \\
& \max _{1 \leq k \leq K_{j}}\left|\mathcal{L}_{j, k}^{\text {inter }, \ell}-\overline{\mathcal{L}}_{j, k}^{\text {inter }, \ell}\right| \underset{K \rightarrow \infty}{\stackrel{\text { a.s. }}{\longrightarrow}} 0 .
\end{aligned}
$$

Proof: The proof relies on the same techniques used in Appendix B and is thus omitted.

Corollary 3. When the channel is perfectly known at all BSs $\left(\tau_{j, \ell, k}=0, \forall j, \ell, k\right)$, we have then :

$$
\begin{aligned}
& \bar{S}_{j, k}^{\mathrm{co}}=\frac{P_{j} \alpha_{j, j, k}}{c_{j}}\left(1-\frac{1}{\tilde{c}_{j}} \tilde{\vartheta}_{j}^{\mathrm{co}}\left(t_{\mathrm{co}}\right)\right) \\
& \overline{\mathcal{L}}_{j, k}^{\mathrm{intra}}=\frac{1}{K_{j}} \sum_{\substack{i=1 \\
i \neq k}}^{K_{j}} \frac{P_{j} \alpha_{j, j, i}}{\left(1+\frac{1}{c_{j} \rho \sigma^{2}} P_{j} \alpha_{j, j, i} \tilde{e}_{j}^{\mathrm{co}}\left(t_{\mathrm{co}}\right)\right)^{2}}, \\
& \overline{\mathcal{L}}_{j, k}^{\text {inter }, \ell}=\frac{1}{K_{j}} \sum_{i=1}^{K_{\ell}} \frac{P_{j} \alpha_{j, \ell, i}}{\left(1+\frac{\beta_{j}}{c_{j} \rho \sigma^{2}} P_{j} \alpha_{j, \ell, i} \tilde{e}_{j}^{\mathrm{co}}\left(t_{\mathrm{co}}\right)\right)^{2}} .
\end{aligned}
$$

Again, we shall note that our asymptotic analysis allow to capture the impact of the large scale parameters on the SLNR performances. In this respect, a close inspection of (54) and (55) reveals that curiously, $\sum_{\ell=1}^{L} \overline{\mathcal{L}}_{j, k}^{\text {inter }, \ell}, \overline{\mathcal{L}}_{j, k}^{\text {intra }}$ which represents the amount of interference leakage generated by the communication between $\mathrm{BS}_{j}$ and UE $k$ to UEs in the other cells and those in the same serving cell is asymptotically the same for all UEs $k$ in cell $j$. The reason behind this result lies in the preocding vector $\mathbf{w}_{j, k}$ being of norm 1 , which makes the $\overline{\mathcal{L}}_{j, k}^{\text {inter, } \ell}$ independent of the channel statistics of UE $k$. Plus, one should note that this property is independent of the type of precoding being used. In fact, we can prove that this result is also valid for ZFBF when normalized to 1 by deriving its interference leakage expressions. Following the same steps as before, we find:

$$
\begin{gathered}
{\left[\overline{\mathcal{L}}_{j, k}^{\text {intra }}\right]_{\mathrm{ZF}}=\sum_{\substack{i=1 \\
i \neq k}}^{K_{j}} \frac{P_{j} \alpha_{j, j, i}}{K_{j}} \tau_{j, j, i}^{2},} \\
{\left[\overline{\mathcal{L}}_{j, k}^{\text {inter }, \ell}\right]=\sum_{i=1}^{K_{\ell}} \frac{P_{j} \alpha_{j, \ell, i}}{K_{j}} \tau_{j, \ell, i}^{2} .}
\end{gathered}
$$

which are both independent of the channel statistics of UE $k$.

\section{Setting of the Coordination Factor $\beta_{j}$}

As explained above, low CSI levels highly lessen the performances of coordinated beamforming which makes the value of the coordination variables $\beta_{j}$ of crucial importance in the design of the coordinated precoding scheme. In this section, we determine the optimal quantities $\beta_{j}^{*}$ that help prevent the degradation that arises when the channel is poorly estimated by maximizing the asymptotic equivalent of SLNR ${ }^{\text {co }}$. However prior to presenting the optimization problem, we first explain the motivation behind maximizing $\overline{\mathrm{SLNR}}^{\mathrm{co}}$ instead of the sum-rate.

To optimize the coordination factor $\beta_{j}$, one could consider the selection of the factor $\beta_{j}$ that maximizes the asymptotic average per UE rate, i.e,

$$
\beta_{1}^{*}, \cdots, \beta_{L}^{*}=\arg \max _{\beta_{1}, \cdots, \beta_{L}} \frac{1}{L} \sum_{\ell=1}^{L} \frac{1}{K_{\ell}} \sum_{k=1}^{K_{\ell}} \log _{2}\left(1+\overline{\operatorname{SINR}}_{\ell, k}\right)
$$

However, this approach has several drawbacks. First, the optimization involves $L$ variables $\left\{\beta_{j}\right\}_{j=1}^{L}$ and thus characterizing the optimal values is not an easy task. Second, optimization could not be performed in a decentralized way as it should assume that all BSs share their large scale statistics with a central node that takes care of the evaluation of the coordination factors $\beta_{j}$.

To overcome these issues, we propose in the sequel a decentralized approach in which each BS selects independently its own coordination factor. More specifically, we propose in our approach that each BS selects the coordination factor that maximizes the asymptotic $\overline{\operatorname{SLNR}}_{j, k}=\frac{S_{j, k}^{\text {co }}}{\overline{\mathcal{L}}_{j, k}^{\text {intra }}+\sum_{\ell=1, \ell \neq j}^{L} \overline{\mathcal{L}}_{j, k}^{\text {inter }, \ell}+\rho \sigma^{2}}$. Note that this value is the same for all $\overline{\mathrm{SLNR}}_{j, k}, k=1, \cdots, K$. This is because as pointed out earlier the interference leakage is the same for all UEs in cell $j$, while the signal terms $\left\{\bar{S}_{j, k}\right\}_{k=1}^{K_{j}}$ are equal up to a constant term independent of $\beta_{j}$. More formally, we propose to select the coordination level factor that solves the following optimization problem:

$$
\begin{gathered}
\beta_{j}^{*}=\underset{\beta_{j}}{\operatorname{argmax}} \overline{\operatorname{SLNR}}_{j, k}, \\
\text { s.t. } \quad 0<\beta_{j}<1 .
\end{gathered}
$$

We note that in the perfect CSI case, the factor $\beta_{j}$ solving (P2) should be equal to 1 as this coincides with the value that maximizes the instantaneous SLNR in (13). Thence, our approach is relevant only in the imperfect CSI case. We expect that as the channel estimation quality deteriorates, the factor $\beta_{j}$ solving (P2) should, in turn, decrease, unveiling the unreliability of the channel estimates used in coordination.

Using some basic mathematical manipulations ${ }^{4}$, we can show that the coordination level $\beta_{j}$ solving (P2) is solution to the following fixed-point equation:

$$
\beta_{j}^{*}=\frac{\mathrm{I}_{j}\left(\tilde{e}_{j}^{\mathrm{co}}\left(t_{\mathrm{co}}\right) \beta_{j}^{*}\left(e_{j}^{\mathrm{co}}\left(t_{\mathrm{co}}\right)\right)^{\prime}-1\right)}{\left(\frac{\left(\bar{S}_{j, k}^{\mathrm{co}}\right)^{\prime}}{\bar{S}_{j, k}^{\mathrm{co}}} \overline{\mathcal{L N}}_{j, k}^{\mathrm{co}}-\left(\overline{\mathcal{L}}_{j, k}^{\mathrm{intra}}\right)^{\prime}\right)\left(\tilde{e}_{j}^{\mathrm{co}}\left(t_{\mathrm{co}}\right) \beta_{j}^{*}\right)^{2}},
$$

${ }^{4}$ Maximizing a fraction $\frac{N}{D}$, where $N \neq 0$ and $D \neq 0$ can be achieved by solving the equation $\frac{N^{\prime}}{N}-\frac{D^{\prime}}{D}=0$. 


$$
\begin{aligned}
& \mathrm{I}_{j}=\frac{2\left(\tilde{c}_{j} t_{\mathrm{co}}\right)^{2}}{K_{j}} \sum_{\substack{\ell=1 \\
\ell \neq j}}^{L} \sum_{i=1}^{K_{\ell}} \frac{\left(1-\tau_{j, \ell, i}^{2}\right)}{P_{j} \alpha_{j, \ell, i}}\left(\Psi_{j, \ell, i}^{\mathrm{co}}\left(t_{\mathrm{co}}, \tilde{c}_{j}\right)\right)^{3} . \\
& \left(e_{j}^{\mathrm{co}}\left(t_{\mathrm{co}}\right)\right)^{\prime}=\frac{\tilde{c}_{j}}{K} \sum_{\substack{\ell=1 \\
\ell \neq j}}^{L} \sum_{i=1}^{K_{\ell}} \frac{\left(\Psi_{j, \ell, i}^{\mathrm{co}}\left(t_{\mathrm{co}}, \tilde{c}_{j}\right)\right)^{2}}{P_{j} \alpha_{j, \ell, i}} \\
& \left(\beta_{j}^{*} \tilde{e}_{j}^{\mathrm{co}}\left(t_{\mathrm{co}}\right)\right)^{2}
\end{aligned}
$$

such that $\mathrm{I}_{j},\left(e_{j}^{\mathrm{co}}\left(t_{\mathrm{co}}\right)\right)^{\prime},\left(\bar{S}_{j, k}^{\mathrm{co}}\right)^{\prime}, \overline{\mathcal{L N}}_{j, k}^{\mathrm{co}}$ and $\left(\mathcal{L}_{j, k}^{\text {intra }}\right)^{\prime}$ are given in (62)-(66), respectively.

\section{NuMERICAL RESUlTS}

In this section, we use Monte Carlo simulations with 10000 channel realizations to validate, for finite system dimensions, the asymptotic results provided in Theorems 1,2 and 3 as well as the expression of optimal $\beta^{*}$. To this end, we consider a two-tier HetNet with one MBS and two mBSs. For both types of BSs, the channel attenuation $\alpha_{j, \ell, k}$ follows the same model as in [29] :

$$
\alpha_{j, \ell, k}=\left(\frac{1}{d_{j, \ell, k}}\right)^{\eta_{j}} \Phi_{j, \ell, k},
$$

where $d_{j, \ell, k}$ is the distance between the serving $\mathrm{BS}_{j}$ and the relevant $\mathrm{UE}_{j, \ell, k}$. The exponent $\eta_{j}$ accounts for the pathloss and $\Phi_{j, \ell, k}$ represents the correlated shadow fading.

Unless otherwise specified, we consider the following network configuration: The macrocell is equipped with $M_{\text {macro }}=$ 256 antennas and $K_{\text {macro }}=80$ users and covers a circular area of radius $R_{\text {macro }}=250 \mathrm{~m}$; the available power at the MBS is set to $P_{1}=46 \mathrm{dBm}$ with a pathloss exponent $\eta_{1}=4$ and correlated shadow fading generated according to [30, equation.1] with standard deviation $\sigma_{\Phi_{1}}^{2}=8 \mathrm{~dB}$; as for microcells, we set $M_{\text {micro }}=16$ and $K_{\text {micro }}=5$ with coverage area of radius $R_{\text {micro }}=35 \mathrm{~m}$; the transmit power of the $\mathrm{mBSs}$ is fixed at $30 \mathrm{dBm}$ and the channel attenuation is configured with $\eta_{j}=3.5$ and $\sigma_{\Phi_{j, \ell, k}}^{2}=8 \mathrm{~dB}, j \neq 1$. To prevent the cells from overlapping, we fix a minimum separation distance of $2 R_{\text {micro }}$ between the BSs. We also set guard distances between the UEs and their serving BS. Denote by $\mathrm{d}_{\min }$ and $\mathrm{d}_{\max }$ the closest and the furthest possible user distances from the BS, respectively. For the MBS, we choose $\mathrm{d}_{\min }=40 \mathrm{~m}$ and $\mathrm{d}_{\max }=249 \mathrm{~m}$ and

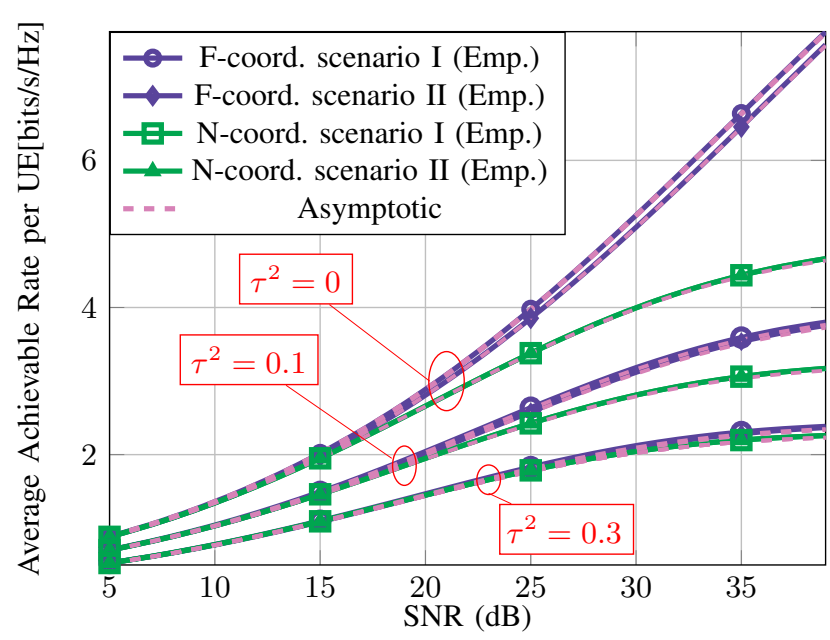

Fig. 2. Macrocell mean per-user sum rate (5) vs. SNR for No, and Full coordination with $\beta_{j}=1, \forall j$ and different levels of CSI.

for the $\mathrm{mBSs}, \mathrm{d}_{\min }=1 \mathrm{~m}$ and $\mathrm{d}_{\max }=25 \mathrm{~m}$. In all simulations, we choose for SLNR based precoding $\rho=1$. We also impose the same quality of channel estimate for all UEs in the network, i.e., $\tau_{j, \ell, i}=\tau, \forall(j, \ell, i)$. In all illustrations, we evaluate the performances of the MBs and one mBS in terms of the average achievable rate per UE defined in (5) and its deterministic equivalent $\bar{R}_{j}=\frac{1}{K_{j}} \sum_{k=1}^{K_{j}} \mathbb{E}\left[\log _{2}\left(1+\overline{\operatorname{SINR}}_{j, k}\right)\right]$.

We propose in Fig. 2 and Fig. 3 to compare the average achievable rate per UE when all BSs employ coordination (F-coord. with $\beta_{j}=1$ ) and when they do not coordinate (N-coord.), for different channel estimations qualities in two scenarios of precoding:

- Scenario I : The macro BS performs SLNR based 


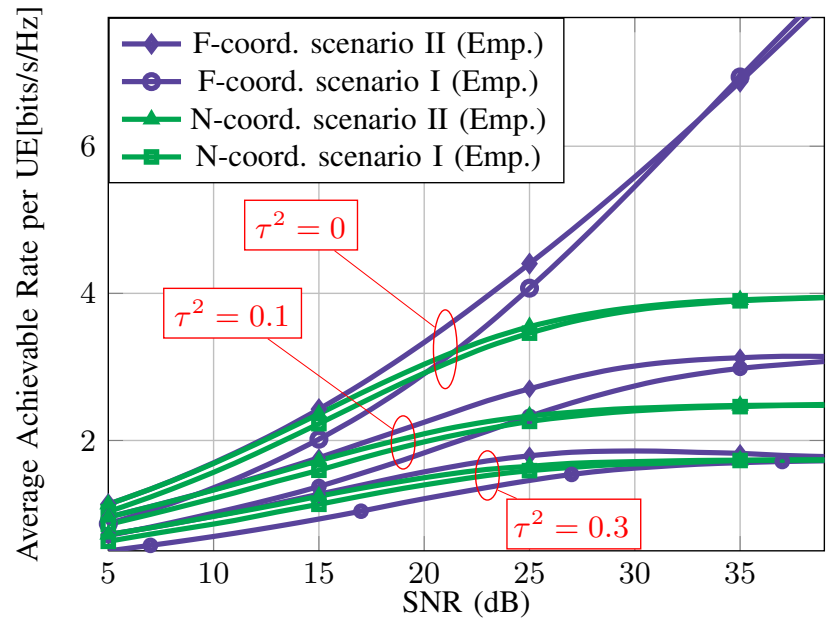

Fig. 3. Microcell mean per-user sum rate (5) vs. SNR for No, and Full coordination with $\beta_{j}=1, \forall j$ and different levels of CSI.

precoding while the micro BSs use ZFBF.

- Scenario II: SLNR based precoding schemes used at all BSs.

The dashed lines represent the deterministic equivalent of each curve. As seen, the simulation results perfectly match the theoretical results provided by Theorems 1 and 2 . This validates the theoretical analysis of this work. Moreover, as seen in Fig. 3, the SLNR-based precoding outperforms the ZFBF especially when coordination is applied. Furthermore, as expected, in the perfect CSI case, coordination enhances the performances of both types of cells and precoding designs. This can be deduced from the noteworthy increase in the SINR in both figures for and both scenarios $\tau^{2}=0$. However, as explained in section III, having a good CSI quality is crucial to the wellfunctioning of coordination. This can be essentially observed when investigating the performance results for $\tau^{2}=0.1$ and particularly $\tau^{2}=0.3$ where the performances of coordination are very close to non-coordinated $\mathrm{BF}$ (even less than noncoordinated $\mathrm{BF}$ for $\mathrm{ZFBF}$ ). These results clearly illustrate the inefficiency of coordination especially in high SNR levels in which the use of coordination incur a loss in performances.

These facts bring us to investigate the performances of coordination with optimal values of $\beta_{j}$ in imperfect CSI scenarios. However, since obtaining $\beta_{j}^{*}$ relies substantially on the SLNR,(P2), we first validate the accuracy of its deterministic equivalent. Towards this aim, we illustrate in Fig.4 SLNR ${ }^{\text {co }}$ (13) and its asymptotic approximation given in Theorem 3 , $\left(\beta_{j}=1, \forall j\right)$, for different levels of CSI at the macrocell. This figure clearly confirms the accuracy of our derivations for finite size networks.

For imperfect CSI levels, we analyze in Fig. 5 and Fig. 6 the performances of employing coordination or not for different settings of the factor $\beta_{j}$ including the optimal $\beta_{j}^{*}$ provided in (61). We can see that the best performances are obtained for $\beta_{j}=\beta_{j}^{*}$. In particular, for both levels of CSI and at high SNR, it can be seen that optimal coordination outperforms the non-coordinated scenario, the coordination with an arbitrary choice of $\beta$ fixed, $\forall \tau$, and the full-coordinated (conventional

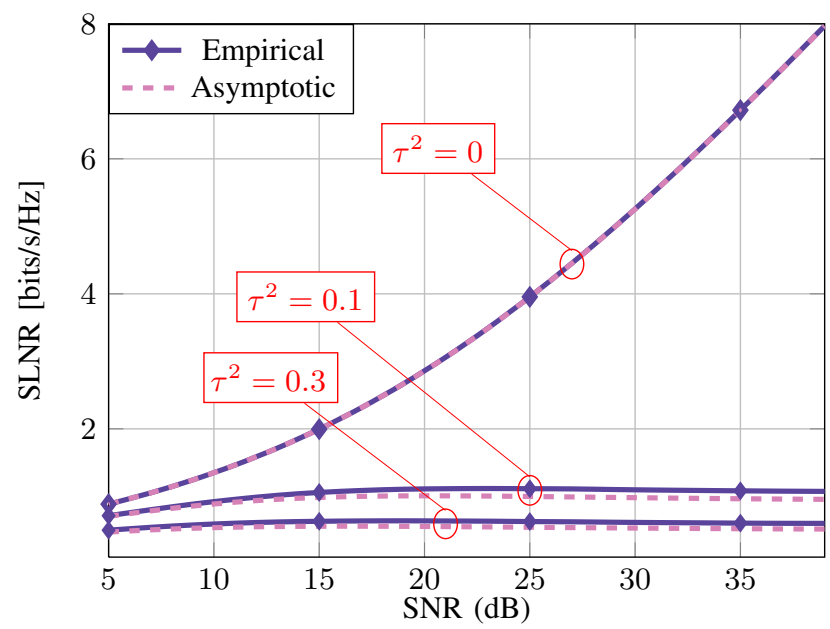

Fig. 4. Macrocell - Accuracy of Asymptotic SLNR (13) with $\beta_{j}=1, \forall j$ and different levels of CSI.

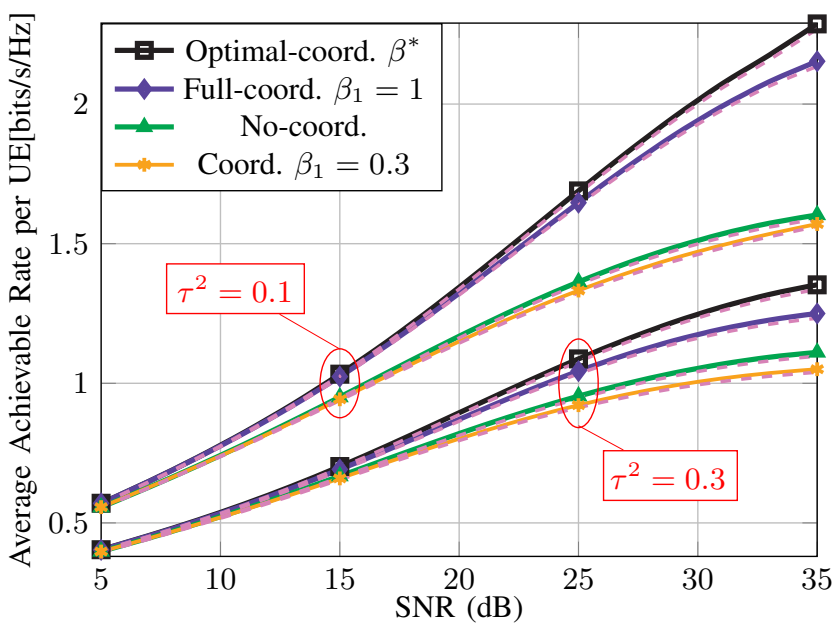

Fig. 5. Macrocell mean per-user sum rate (5) vs. SNR for No, and Full coordination and Optimal Coordination for different levels of CSI. Solid and dashed lines represent empirical and asymptotic results, respectively.

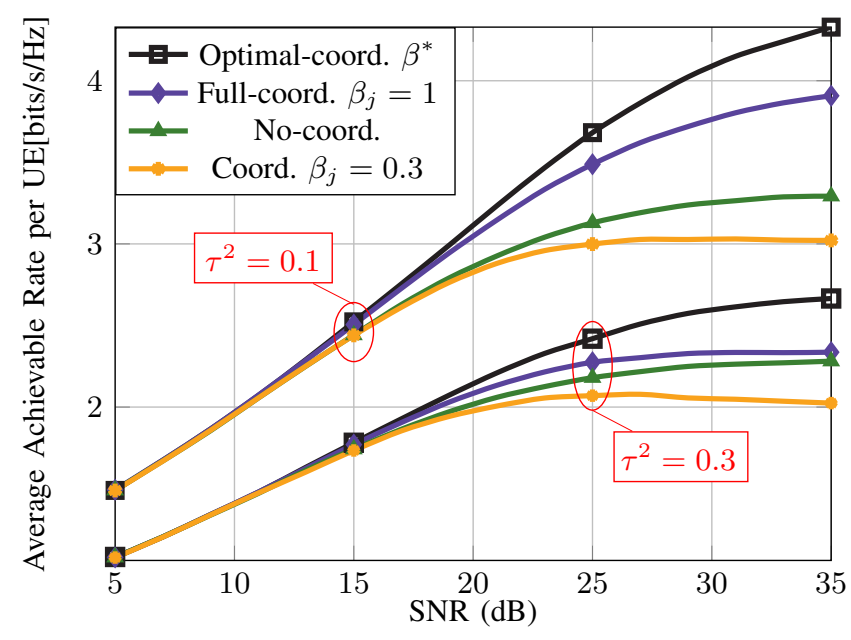

Fig. 6. Microcell mean per-user sum rate (5) vs. SNR for No, and Full coordination and Optimal Coordination for different levels of CSI. 


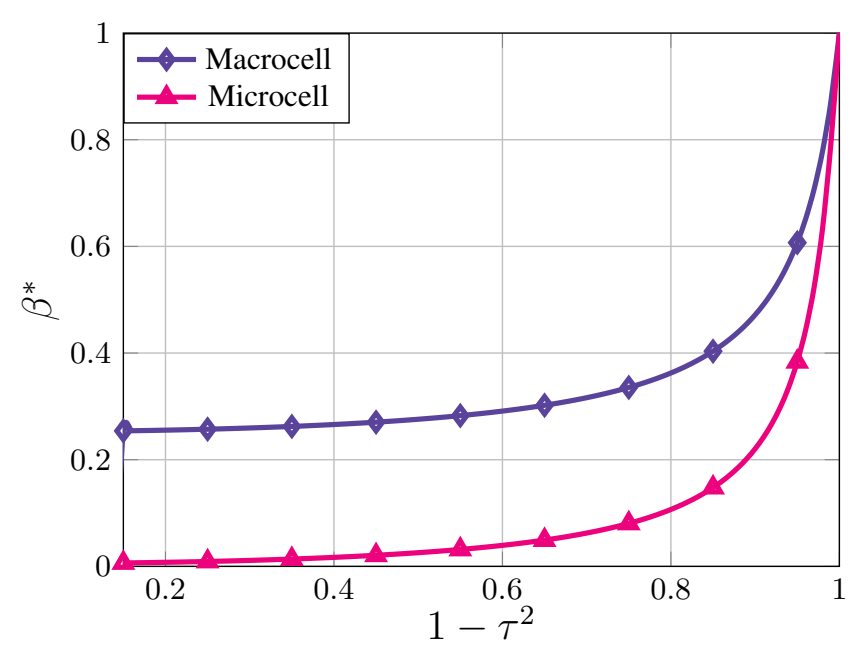

Fig. 7. Macrocell and microcell $\beta^{*}$ Vs. quality of CSI $\left(1-\tau^{2}\right)$, SNR=10dB.

SLNR based precoding) with $\beta_{j}=1, \forall j$. Furthermore, for very poor channel estimation qualities, optimal coordination converges, understandably, towards the non-coordinated cases, i.e. $\beta^{*} \rightarrow 0$. It is also important to note that the perfect CSI curves are not included in these figures since, for $\tau^{2}=0$, $\beta_{j}^{*}=1$, which leads to the same plots as in figures 2 and 3 . Accordingly and as we mentioned in section III-C, the provided optimal coordination factor in (61) depends highly on the level of available CSI. Obviously, $\beta^{*}$ takes higher values with the increase of the level of CSI which is depicted by $\left(1-\tau^{2}\right)$ and clearly shown in Fig.7.

\section{Conclusions}

In this work, we analysed interference mitigation in large scale two-tier HetNets. We investigate the efficiency of coordinated beamforming compared to non-coordinated processing assuming imperfect CSI levels. Large-scale random matrix theory tools were used to develop closed form expressions of the SINR and SLNR quantities. A selection of numerical results shows that these results are accurate for finite system dimension, albeit computed assuming the asymptotic regime. Throughout this study, we demonstrated in what manner coordination enhances the performances and showed how the level of CSI impacts its efficiency. To remedy this issue, we proposed a modified design of the SLNR based precoding, in which we introduce a normalizing factor $\beta$. The value of this latter is obtained via the maximization of the network utility, SLNR, taking into account the quality of CSI. The precoding design we obtain is shown to outperform both non-coordinated and conventional coordinated SLNR based precoding. In this paper, the system model includes a finite number of micro-cells and one macro-cell. Each $\mathrm{BS}_{j}$ would use the same level of coordination $\beta_{j}^{*}$ with all the other BSs. For future work, an interesting approach would be to extend the optimization to include individual optimizations, i.e. $\beta_{j, \ell}^{*}$, and study the impact of densification on the coordination.

\section{APPENDIX A}

\section{IMPORTANT RESULTS FROM RANDOM MATRIX THEORY}

Our work belongs to the lineage of recent studies investigating the peroformances of large dimensional systems using random matrix theory tools [25]. For a better understanding of the asymptotic results derived in this paper and the proofs given in the subsequent sections, we provide in this appendix the necessary lemmas and propositions used for the derivations. Throughout this appendix $\mathbf{H}$ denotes a $M \times K$ complex matrix satisfying the following assumption

\section{Assumption 3.}

$$
\mathbf{H}=\frac{1}{\sqrt{K}} \mathbf{X} \tilde{\mathbf{D}}^{\frac{1}{2}}
$$

where $\mathbf{X} \in \mathbb{C}^{M \times K}$ is a standard Gaussian matrix with zero mean and unit variance and $\tilde{\mathbf{D}}$ is a $K \times K$ diagonal matrix.

We further consider the following assumptions:

Assumption 4. The diagonal elements of $\tilde{\mathbf{D}}$ verify

$$
0<\liminf \sum_{i=1}^{K} \tilde{d}_{i}<\limsup \sum_{i=1}^{K} \tilde{d}_{i}<\infty .
$$

Assumption 5. Let $c=\frac{K}{M}$. Then, we have:

$$
0<\liminf c<\lim \sup c<\infty .
$$

We review results concerning the behavior of quantities involving $\mathbf{H}$ in the asymptotic regime defined in Assumption 5. These results will be extensively used in the proof of Theorems 1 and 2.

Lemma 1 (Inversion Lemma). [31, Lemma 2.2] Let $\mathbf{H}$ be an $M \times K$ complex matrix and $\mathbf{h}_{k} \in \mathbb{C}^{M \times 1}$ its $k$-th column. Denote by $\mathbf{H}_{k}$, the matrix $\mathbf{H}$ without $\mathbf{h}_{k}$. For $t \in \mathbb{C}$, we define the resolvent matrices associated with $\mathbf{H}$ and $\mathbf{H}_{k}$ as: $\mathbf{Q}(t)=\left(\frac{t}{K} \mathbf{H H}^{H}+\mathbf{I}_{M}\right)^{-1}$ and $\mathbf{Q}_{k}(t)=\left(\frac{t}{K} \mathbf{H}_{k} \mathbf{H}_{k}^{H}+\mathbf{I}_{M}\right)^{-1}$. It follows that:

$$
\mathbf{Q}(t) \mathbf{h}_{k}=\frac{\mathbf{Q}_{k}(t) \mathbf{h}_{k}}{1+\frac{t}{K} \mathbf{h}_{k}^{H} \mathbf{Q}_{k}(t) \mathbf{h}_{k}}
$$

and

$$
\mathbf{Q}(t)=\mathbf{Q}_{k}(t)-\frac{\frac{t}{K} \mathbf{Q}_{k}(t) \mathbf{h}_{k} \mathbf{h}_{k}^{H} \mathbf{Q}_{k}(t)}{1+\frac{t}{K} \mathbf{h}_{k}^{H} \mathbf{Q}_{k}(t) \mathbf{h}_{k}} .
$$

Corollary 4. Let $\mathbf{h}, \mathbf{h}_{k}, \mathbf{Q}(t)$ and $\mathbf{Q}_{k}(t)$ be defined as in Lemma 1. We have then :

$$
\frac{\mathbf{Q}(t) \mathbf{h}_{k}}{\left\|\mathbf{Q}(t) \mathbf{h}_{k}\right\|}=\frac{\mathbf{Q}_{k}(t) \mathbf{h}_{k}}{\left\|\mathbf{Q}_{k}(t) \mathbf{h}_{k}\right\|} .
$$

Lemma 2 (Convergence of quadratic forms). [32] Let $\mathbf{A}_{\mathrm{M}} \in$ $\mathbb{C}^{M \times M}$ be a sequence of matrices with bounded spectral norms, i.e. $\lim \sup _{\mathrm{M}}\|A\|_{2}<\infty$. Let $\mathrm{x}_{\mathrm{M}, 1}, \mathrm{x}_{\mathrm{M}, 2}, \ldots$, with $\mathrm{x}_{\mathrm{M}, \mathrm{K}} \in$ $\mathbb{C}^{M \times 1}$ be $K$ random vectors with i.i.d complex Gaussian entries and variance $\frac{1}{M}$ and independent from $\mathbf{A}_{M}$. It holds that, as $M$ and $K$ grow to inifnity together

$$
\mathbf{x}_{\mathrm{M}, \mathrm{k}}^{H} \mathbf{A}_{\mathrm{M}} \mathbf{x}_{\mathrm{M}, \mathrm{k}}-\frac{1}{M} \operatorname{tr}\left(\mathbf{A}_{\mathrm{M}}\right) \underset{M \rightarrow \infty}{\stackrel{a . s .}{\longrightarrow}} 0 .
$$


We also have

$$
\max _{1 \leq k \leq K}\left|\mathbf{x}_{\mathrm{M}, \mathrm{k}}^{H} \mathbf{A}_{M} \mathbf{x}_{\mathrm{M}, \mathrm{k}}-\frac{1}{M} \operatorname{tr}\left(\mathbf{A}_{M}\right)\right| \underset{M, K \rightarrow \infty}{\stackrel{\text { a.s. }}{\longrightarrow}} 0 .
$$

Corollary 5. Let $\mathbf{A}_{\mathrm{M}} \in \mathbb{C}^{M \times M}$ be defined as in Lemma 2 . Let $\mathrm{x}_{\mathrm{M}}$ and $\mathrm{y}_{\mathrm{M}}$ be $M \times 1$ mutually independent vectors with i.i.d complex Gaussian entries and variance $\frac{1}{M}$. Then,

$$
\mathbf{x}_{\mathrm{M}}^{H} \mathbf{A}_{\mathrm{M}} \mathbf{y}_{\mathrm{M}} \underset{M \rightarrow \infty}{\stackrel{\text { a.s. }}{\longrightarrow}} 0 .
$$

Lemma 3 (Rank one perturbation ). [33, Lemma 14.3] Let $\mathbf{Q}(t)$ and $\mathbf{Q}_{k}(t)$ be defined as in Lemma 1. Then, for any sequence of matrices $\left(\mathbf{A}_{\mathbf{M}}\right)$ with bounded spectral norm, we have:

$$
\frac{1}{M} \operatorname{tr}\left(\mathbf{A}_{M} \mathbf{Q}(t)\right)-\frac{1}{M} \operatorname{tr}\left(\mathbf{A}_{N} \mathbf{Q}_{k}(t)\right) \underset{M \rightarrow \infty}{\stackrel{\text { a.s. }}{\longrightarrow}} 0 .
$$

In the following, we propose deterministic equivalents approximating quantities $\frac{1}{K} \operatorname{tr}(\mathbf{A Q}(t))$ and $\frac{1}{K} \operatorname{tr}\left(\mathbf{A}(\mathbf{Q}(t))^{2}\right)$, respectively.

Proposition 1 (Deterministic equivalent for $\left.\frac{1}{K} \operatorname{tr}(\mathbf{A Q}(t))\right)$. Let $\mathbf{H}$ be as in Assumption 3. Define $\mathbf{Q}(t)$ to be the resolvent matrix associated with $\mathbf{H}$. Then, under Assumption 4, the following equation:

$$
e(t)=\frac{1}{K} \sum_{i=1}^{K} \frac{\tilde{d}_{i}}{t+\frac{1}{c} \tilde{d}_{i} \tilde{e}(t)},
$$

with $\tilde{e}(t)=\frac{1}{1+e(t)}$ admits a unique solution for $t>0$.

Moreover, for any $t>0$ and under Assumptions 4-5, the following convergence holds true:

$$
\frac{1}{K} \operatorname{tr}(\mathbf{A Q}(t))-t^{-1} \tilde{e}(t) \frac{1}{K} \operatorname{tr}(\mathbf{A}) \underset{K \rightarrow \infty}{\stackrel{a . s .}{\longrightarrow}} 0 .
$$

Proposition 2 (Deterministic equivalent for $\frac{1}{K} \operatorname{tr}\left(\mathbf{A}(\mathbf{Q}(t))^{2}\right)$ ). Consider the setting of Proposition 1.

Define

$$
\Gamma(t, c)=\frac{1}{1+\frac{t c}{\tilde{d}_{i} \tilde{e}(t)}},
$$

and:

$$
\tilde{\vartheta}(t)=\frac{c^{2}}{K} \sum_{i=1}^{K}(\Gamma(t, c))^{2} .
$$

Then, under Assumptions 4 and 5 ,

$$
\begin{aligned}
\frac{1}{K} \operatorname{tr} & \left.\mathbf{A}(\mathbf{Q}(t))^{2}\right) \\
& -\frac{(\tilde{e}(t))^{2}}{t^{2}\left(1-c^{-1} \tilde{\vartheta}(t)\right)} \frac{1}{K} \operatorname{tr} \mathbf{A} \underset{K \rightarrow \infty}{\stackrel{\text { a.s. }}{\longrightarrow}} 0,
\end{aligned}
$$

\section{APPENDIX B}

\section{PROOF OF THEOREMS 1 AND 2}

The objective here is to proof the validity of the deterministic equivalents given in Theorems 1 and 2 . We consider herein the notations and the statistical assumptions given in sections II-III.

\section{A. Proof of Theorem 1}

Define $\widehat{\mathbf{H}}_{j, j}$ as in (9) and let $\widehat{\mathbf{H}}_{j, j_{k}}$ be the matrix obtained after removing its $k$-th column. The resolvent matrices associated with $\widehat{\mathbf{H}}_{j, j}$ and $\widehat{\mathbf{H}}_{j, j_{k}}$ are defined as:

$$
\mathbf{Q}_{j}\left(\rho \sigma^{2}\right)=\left(\widehat{\mathbf{H}}_{j, j} \widehat{\mathbf{H}}_{j, j}^{\mathrm{H}}+\rho \sigma^{2} \mathbf{I}_{M_{j}}\right)^{-1}
$$

and

$$
\mathbf{Q}_{j}^{(\mathrm{k})}\left(\rho \sigma^{2}\right)=\left(\widehat{\mathbf{H}}_{j, j_{k}} \widehat{\mathbf{H}}_{j, j_{k}}^{\mathrm{H}}+\rho \sigma^{2} \mathbf{I}_{M_{j}}\right)^{-1}
$$

respectively.

When no coordination is applied, consider $e_{j}^{\text {no-co }}\left(\rho \sigma^{2}\right)$, $\tilde{e}_{j}^{\text {no-co }}\left(\rho \sigma^{2}\right)$ and $\tilde{\vartheta}_{j}^{\text {no-co }}\left(\rho \sigma^{2}\right)$ defined in (22) and (23) and (25). As per Propositions 1 and 2, for any sequence of matrices $\mathbf{A}_{M}$ with bounded spectral norm, the following convergences hold true:

$$
\begin{aligned}
& \frac{1}{K_{j}} \operatorname{tr}\left(\mathbf{A}_{M} \mathbf{Q}_{j}\left(\rho \sigma^{2}\right)\right) \\
& \quad-\frac{1}{\rho \sigma^{2}} \tilde{e}_{j}^{\text {no-co }}\left(\rho \sigma^{2}\right) \frac{1}{K_{j}} \operatorname{tr}\left(\mathbf{A}_{M}\right) \underset{K \rightarrow \infty}{\stackrel{a . s .}{\longrightarrow}} 0,
\end{aligned}
$$

and

$$
\begin{aligned}
& \frac{1}{K_{j}} \operatorname{tr}\left(\mathbf{A}_{M}\left(\mathbf{Q}_{j}\left(\rho \sigma^{2}\right)\right)^{2}\right) \\
& -\frac{\left(\tilde{e}_{j}^{\mathrm{no}-\mathrm{co}}\left(\rho \sigma^{2}\right)\right)^{2}}{\left(\rho \sigma^{2}\right)^{2}\left(1-\frac{1}{c_{j}} \tilde{\vartheta}_{j}^{\mathrm{no}-\mathrm{co}}\left(\rho \sigma^{2}\right)\right)} \frac{1}{K_{j}} \operatorname{tr}\left(\mathbf{A}_{M}\right) \underset{K \rightarrow \infty}{\stackrel{a . s .}{\longrightarrow}} 0,
\end{aligned}
$$

1) Deterministic Equivalent for $S_{j, k}^{\mathrm{no}-\mathrm{co}}$ : First, we write $S_{j, k}^{\mathrm{no}-\mathrm{co}}$ as:

$$
\begin{aligned}
S_{j, k}^{\mathrm{no}-\mathrm{co}} & =\left|\mathbf{h}_{j, j, k}^{\mathrm{H}} \widehat{\mathbf{w}}_{j, k}^{\mathrm{no}-\mathrm{co}}\right|^{2} \\
& =\frac{\left|\mathbf{h}_{j, j, k}^{\mathrm{H}} \mathbf{Q}_{j}\left(\rho \sigma^{2}\right) \widehat{\mathbf{h}}_{j, j, k}\right|^{2}}{\widehat{\mathbf{h}}_{j, j, k}^{\mathrm{H}}\left(\mathbf{Q}_{j}\left(\rho \sigma^{2}\right)\right)^{2} \widehat{\mathbf{h}}_{j, j, k}}
\end{aligned}
$$

Using Corollary 4 , we can substitute $\mathbf{Q}_{j}(t)$ by $\mathbf{Q}_{j}^{(\mathrm{k})}(t)$, thus yielding:

$$
S_{j, k}^{\mathrm{no}-\mathrm{co}}=\frac{\left|\mathbf{h}_{j, j, k}^{\mathrm{H}} \mathbf{Q}_{j}^{(\mathrm{k})}\left(\rho \sigma^{2}\right) \widehat{\mathbf{h}}_{j, j, k}\right|^{2}}{\widehat{\mathbf{h}}_{j, j, k}^{\mathrm{H}}\left(\mathbf{Q}_{j}^{(\mathrm{k})}\left(\rho \sigma^{2}\right)\right)^{2} \widehat{\mathbf{h}}_{j, j, k}} .
$$

For simplicity, we derive the deterministic equivalents of the numerator and denominator separately. We start by the numerator $\mathbf{h}_{j, j, k}^{\mathrm{H}} \mathbf{Q}_{j}^{(\mathrm{k})}\left(\rho \sigma^{2}\right) \widehat{\mathbf{h}}_{j, j, k}$. Due to the real channel being independent of the estimation error (8), a direct application of Lemmas 2 and 5 implies that:

$$
\begin{aligned}
\mathbf{h}_{j, j, k}^{\mathrm{H}} & \mathbf{Q}_{j}^{(\mathrm{k})}\left(\rho \sigma^{2}\right) \widehat{\mathbf{h}}_{j, j, k} \\
& -\frac{P_{j} \alpha_{j, j, k}}{K_{j}}\left(1-\tau^{2}\right) \operatorname{tr}\left(\mathbf{Q}_{j}^{(\mathrm{k})}\left(\rho \sigma^{2}\right)\right) \underset{K \rightarrow \infty}{\stackrel{a . s .}{\longrightarrow}} 0 .
\end{aligned}
$$


Finally, using the rank-one perturbation Lemma (Lemma 3) along with (80), we ultimately obtain:

$$
\begin{aligned}
\mathbf{h}_{j, j, k}^{\mathrm{H}} & \mathbf{Q}_{j}^{(k)}\left(\rho \sigma^{2}\right) \widehat{\mathbf{h}}_{j, j, k} \\
& -P_{j} \alpha_{j, j, k} \frac{\left(1-\tau^{2}\right)}{c_{j} \rho \sigma^{2}} \tilde{e}_{j}^{\text {no-co }}\left(\rho \sigma^{2}\right) \underset{K \rightarrow \infty}{\stackrel{a . s .}{\longrightarrow}} 0 .
\end{aligned}
$$

Similarly, a deterministic equivalent of the denominator $\widehat{\mathbf{h}}_{j, j, k}^{\mathrm{H}}\left(\mathbf{Q}_{j}^{(k)}\left(\rho \sigma^{2}\right)\right)^{2} \widehat{\mathbf{h}}_{j, j, k}$ can be obtained using again the convergence in Lemma 2 along with the rank-one perturbation Lemma, thus yielding:

$\widehat{\mathbf{h}}_{j, j, k}^{\mathrm{H}}\left(\mathbf{Q}_{j}^{(k)}\left(\rho \sigma^{2}\right)\right)^{2} \widehat{\mathbf{h}}_{j, j, k}-\frac{P_{j} \alpha_{j, j, k}}{K_{j}} \operatorname{tr}\left(\left(\mathbf{Q}_{j}\left(\rho \sigma^{2}\right)\right)^{2}\right) \underset{K \rightarrow \infty}{\stackrel{a . s .}{\longrightarrow}} 0$.

Finally, invoking (81), we obtain:

$$
\begin{aligned}
& \widehat{\mathbf{h}}_{j, j, k}^{\mathrm{H}}\left(\mathbf{Q}_{j}^{(k)}\left(\rho \sigma^{2}\right)\right)^{2} \widehat{\mathbf{h}}_{j, j, k} \\
& \quad-P_{j} \alpha_{j, j, k} \frac{\left(\tilde{e}_{j}^{\mathrm{no}-\mathrm{co}}\left(\rho \sigma^{2}\right)\right)^{2}}{c_{j}\left(\rho \sigma^{2}\right)^{2}\left(1-\frac{1}{c_{j}} \tilde{\vartheta}_{j}^{\mathrm{no}-\mathrm{co}}(\rho \sigma)\right)} \frac{a . s .}{K \rightarrow \infty} 0 .
\end{aligned}
$$

2) Deterministic Equivalent for $I_{j, k}^{\text {intra,no-co }}$ : To begin with, we define $\mathbf{D}$ and $\overline{\mathbf{D}}$ as:

$$
\mathbf{D}=\operatorname{diag}\left\{\left(\widehat{\mathbf{h}}_{j, j, i}^{\mathrm{H}}\left(\mathbf{Q}_{j}\left(\rho \sigma^{2}\right)\right)^{2} \widehat{\mathbf{h}}_{j, j, i}\right)^{-1}\right\}_{i=1}^{K_{j}}
$$

and

$$
\overline{\mathbf{D}}=\operatorname{diag}\left\{\frac{\left(1+\frac{P_{j} \alpha_{j, j, i}}{K_{j}} \operatorname{tr}\left(\mathbf{Q}_{j}\left(\rho \sigma^{2}\right)\right)\right)^{2}}{\frac{P_{j} \alpha_{j, j, i}}{K_{j}} \operatorname{tr}\left(\mathbf{Q}_{j}^{2}\left(\rho \sigma^{2}\right)\right)}\right\}_{i=1}^{K_{j}}
$$

We express $I_{j, k}^{\text {intra,no-co }}$ as:

$$
\begin{aligned}
I_{j, k}^{\text {intra,no-co }} & =\sum_{\substack{i=1 \\
i \neq k}}^{K_{j}}\left|\mathbf{h}_{j, j, k}^{\mathrm{H}} \widehat{\mathbf{w}}_{j, i}^{\text {no-co }}\right|^{2} \\
& =\sum_{\substack{i=1 \\
i \neq k}}^{K_{j}} \frac{\mathbf{h}_{j, j, k}^{\mathrm{H}} \mathbf{Q}_{j}\left(\rho \sigma^{2}\right) \widehat{\mathbf{h}}_{j, j, i} \widehat{\mathbf{h}}_{j, j, i}^{\mathrm{H}} \mathbf{Q}_{j}\left(\rho \sigma^{2}\right) \mathbf{h}_{j, j, k}}{\widehat{\mathbf{h}}_{j, j, i}^{\mathrm{H}}\left(\mathbf{Q}_{j}\left(\rho \sigma^{2}\right)\right)^{2} \widehat{\mathbf{h}}_{j, j, i}} \\
& =\mathbf{h}_{j, j, k}^{\mathrm{H}} \mathbf{Q}_{j}\left(\rho \sigma^{2}\right) \widehat{\mathbf{H}}_{j, j_{k}} \mathbf{D}_{k} \widehat{\mathbf{H}}_{j, j_{k}}^{\mathrm{H}} \mathbf{Q}_{j}\left(\rho \sigma^{2}\right) \mathbf{h}_{j, j, k},
\end{aligned}
$$

where $\mathbf{D}_{k}$ is $\mathbf{D}$ with row $k$ and column $k$ being removed. We also form $\overline{\mathbf{D}}_{k}$ by removing the $k$-th row and the $k$-th column of matrix $\overline{\mathbf{D}}$.

Using (72), we obtain:

$$
\left\|\overline{\mathbf{D}}_{k}-\mathbf{D}_{k}\right\| \underset{K \rightarrow \infty}{\stackrel{a . s .}{\longrightarrow}} 0 .
$$

This allows us to approximate $I_{j, k}^{\mathrm{intra}, \mathrm{no}-\mathrm{co}}$ as:

$$
\begin{aligned}
& I_{j, k}^{\text {intra,no-co }} \\
& \quad-\mathbf{h}_{j, j, k}^{\mathrm{H}} \mathbf{Q}_{j}\left(\rho \sigma^{2}\right) \widehat{\mathbf{H}}_{j, j_{k}} \overline{\mathbf{D}}_{k} \widehat{\mathbf{H}}_{j, j_{k}}^{\mathrm{H}} \mathbf{Q}_{j}\left(\rho \sigma^{2}\right) \mathbf{h}_{j, j, k} \underset{K \rightarrow \infty}{\stackrel{a . s .}{\longrightarrow}} 0,
\end{aligned}
$$

For the results of Appendix A to be applicable, we need to handle the dependence of $\mathbf{Q}_{j}\left(\rho \sigma^{2}\right)$ on $\mathbf{h}_{j, j, k}$. One way to achieve that is to use the property given in (69), thereby yielding (88). A careful analysis of this latter shows that we need to obtain deterministic equivalents for the following terms to conclude:

$$
\begin{aligned}
& \text { - } A_{1}\left(\rho \sigma^{2}\right)=\frac{1}{K_{j}} \operatorname{tr}\left(\mathbf{Q}_{j}^{(k)}\left(\rho \sigma^{2}\right) \widehat{\mathbf{H}}_{j, j_{k}} \overline{\mathbf{D}}_{k} \widehat{\mathbf{H}}_{j, j_{k}}^{\mathrm{H}} \mathbf{Q}_{j}^{(k)}\left(\rho \sigma^{2}\right)\right), \\
& \text { - } A_{2}\left(\rho \sigma^{2}\right)=\mathbf{h}_{j, j, k}^{\mathrm{H}} \mathbf{Q}_{j}^{(k)}\left(\rho \sigma^{2}\right) \widehat{\mathbf{h}}_{j, j, k}, \\
& \text { - } A_{3}\left(\rho \sigma^{2}\right)=\widehat{\mathbf{h}}_{j, j, k}^{\mathrm{H}} \mathbf{Q}_{j}^{(\mathrm{k})}\left(\rho \sigma^{2}\right) \widehat{\mathbf{h}}_{j, j, k} .
\end{aligned}
$$

The asymptotic limit of $A_{2}\left(\rho \sigma^{2}\right)$ is already given in (83). As for $A_{3}\left(\rho \sigma^{2}\right)$ a deterministic equivalent unfolds from a direct application of Lemma 2 along with (80), thus yielding:

$$
A_{3}\left(\rho \sigma^{2}\right)-P_{j} \alpha_{j, j, k} \frac{\tilde{e}_{j}^{\mathrm{no}-\mathrm{co}}\left(\rho \sigma^{2}\right)}{c_{j} \rho \sigma^{2}} \underset{K \rightarrow \infty}{\stackrel{a . s .}{\longrightarrow}} 0 .
$$

On the other hand, $A_{1}\left(\rho \sigma^{2}\right)$ has a variance decreasing at a rate $M^{-2}$ [34, Proposition 4], and as such satisfies:

$$
A_{1}\left(\rho \sigma^{2}\right)-\mathbb{E}\left[A_{1}\left(\rho \sigma^{2}\right)\right] \underset{K \rightarrow \infty}{\stackrel{a . s .}{\longrightarrow}} 0,
$$

Now, expanding $\mathbb{E}\left[A_{1}\left(\rho \sigma^{2}\right)\right]$, we obtain:

$$
\mathbb{E}\left[A_{1}\left(\rho \sigma^{2}\right)\right]=\frac{1}{K_{j}} \sum_{\substack{i=1 \\ i \neq k}}^{K_{j}} \bar{d}_{i} \mathbb{E}\left[\operatorname{tr}\left(\widehat{\mathbf{h}}_{j, j, i}^{\mathrm{H}}\left(\mathbf{Q}_{j}^{(k)}\left(\rho \sigma^{2}\right)\right)^{2} \widehat{\mathbf{h}}_{j, j, i}\right)\right] .
$$

Again, the dependence of $\mathbf{Q}_{j}^{(k)}\left(\rho \sigma^{2}\right)$ on $\widehat{\mathbf{h}}_{j, j, i}^{\mathrm{H}}$ can be controlled using (68).

$$
\mathbb{E}\left[A_{1}\left(\rho \sigma^{2}\right)\right]-1 \underset{K \rightarrow \infty}{\stackrel{a . s .}{\longrightarrow}} 0
$$

Plugging the deterministic equivalents of $A_{2}\left(\rho \sigma^{2}\right)$ (83), $A_{3}\left(\rho \sigma^{2}\right)(89)$ and $A_{1}\left(\rho \sigma^{2}\right)$ (92) into (88) yields the asymptotic expression in (93). This latter is excatly the same expression of $\bar{I}_{j, k}^{\text {intra,no-co }}$ given in Theorem 1 once we substitute $\frac{1}{K_{j}} \operatorname{tr}\left(\mathbf{Q}_{j}\left(\rho \sigma^{2}\right)\right)$ and $\frac{1}{K_{j}} \operatorname{tr}\left(\left(\mathbf{Q}_{j}\left(\rho \sigma^{2}\right)\right)^{2}\right)$ by their respective asymptotic limits (80) and (81).

3) Deterministic Equivalent for $I_{j, \ell, k}^{\mathrm{inter,no-co}}$ : To begin with, we write $I_{j, \ell, k}^{\text {inter,no-co }}$ as:

$$
\begin{aligned}
I_{j, \ell, k}^{\text {inter,no-co }} & =\sum_{\substack{i=1 \\
i \neq k}}^{K_{j}}\left|\mathbf{h}_{j, \ell, k}^{\mathrm{H}} \widehat{\mathbf{w}}_{j, i}^{\text {no-co }}\right|^{2} \\
& =\sum_{\substack{i=1 \\
i \neq k}}^{K_{j}} \frac{\mathbf{h}_{j, \ell, k}^{\mathrm{H}} \mathbf{Q}_{j}\left(\rho \sigma^{2}\right) \widehat{\mathbf{h}}_{j, j, i} \widehat{\mathbf{h}}_{j, j, i}^{\mathrm{H}} \mathbf{Q}_{j}\left(\rho \sigma^{2}\right) \mathbf{h}_{j, \ell, k}}{\widehat{\mathbf{h}}_{j, j, i}^{\mathrm{H}}\left(\mathbf{Q}_{j}\left(\rho \sigma^{2}\right)\right)^{2} \widehat{\mathbf{h}}_{j, j, i}}
\end{aligned}
$$

Following the same technicalities leading to (87), we have:

$$
\begin{aligned}
& I_{j, \ell, k}^{\text {inter,no-co }} \\
& \quad-\mathbf{h}_{j, \ell, k}^{\mathrm{H}} \mathbf{Q}_{j}\left(\rho \sigma^{2}\right) \widehat{\mathbf{H}}_{j, j_{k}} \overline{\mathbf{D}}_{k} \widehat{\mathbf{H}}_{j, j_{k}}^{\mathrm{H}} \mathbf{Q}_{j}\left(\rho \sigma^{2}\right) \mathbf{h}_{j, \ell, k} \underset{K \rightarrow \infty}{\stackrel{a . s .}{\longrightarrow}} 0,
\end{aligned}
$$

where $\overline{\mathbf{D}}_{k}$ has the same expression as in (87).

Note that here, the result (92) can be applied directly since $\mathbf{h}_{j, \ell, k}$ is independent of $\mathbf{Q}_{j}\left(\rho \sigma^{2}\right)$, which is in contrast to (88) where lemma 1 had to be used. Therefore, we can easily find that

$$
I_{j, \ell, k}^{\text {inter,no-co }}-P_{j} \alpha_{j, \ell, k} \underset{K \rightarrow \infty}{\stackrel{a . s .}{\longrightarrow}} 0 .
$$




$$
\begin{aligned}
& I_{j, k}^{\text {intra,no-co }}-\mathbf{h}_{j, j, k}^{\mathrm{H}} \mathbf{Q}_{j}^{(\mathrm{k})}\left(\rho \sigma^{2}\right) \widehat{\mathbf{H}}_{j, j_{k}} \overline{\mathbf{D}}_{k} \widehat{\mathbf{H}}_{j, j_{k}}^{\mathrm{H}} \mathbf{Q}_{j}^{(\mathrm{k})}\left(\rho \sigma^{2}\right) \mathbf{h}_{j, j, k} \\
& +\frac{\mathbf{h}_{j, j, k}^{\mathrm{H}} \mathbf{Q}_{j}^{(\mathrm{k})}\left(\rho \sigma^{2}\right) \widehat{\mathbf{H}}_{j, j_{k}} \overline{\mathbf{D}}_{k} \widehat{\mathbf{H}}_{j, j_{k}}^{\mathrm{H}} \mathbf{Q}_{j}^{(\mathrm{k})}\left(\rho \sigma^{2}\right) \widehat{\mathbf{h}}_{j, j, k} \widehat{\mathbf{h}}_{j, j, k}^{\mathrm{H}} \mathbf{Q}_{j}^{(\mathrm{k})}\left(\rho \sigma^{2}\right) \mathbf{h}_{j, j, k}}{1+\widehat{\mathbf{h}}_{j, j, k}^{\mathrm{H}} \mathbf{Q}_{j}^{(\mathrm{k})}\left(\rho \sigma^{2}\right) \widehat{\mathbf{h}}_{j, j, k}} \\
& +\frac{\mathbf{h}_{j, j, k}^{\mathrm{H}} \mathbf{Q}_{j}^{(k)}\left(\rho \sigma^{2}\right) \widehat{\mathbf{h}}_{j, j, k} \widehat{\mathbf{h}}_{j, j, k}^{\mathrm{H}} \mathbf{Q}_{j}^{(k)}\left(\rho \sigma^{2}\right) \widehat{\mathbf{H}}_{j, j_{k}} \overline{\mathbf{D}}_{k} \widehat{\mathbf{H}}_{j, j_{k}}^{\mathrm{H}} \mathbf{Q}_{j}^{(k)}\left(\rho \sigma^{2}\right) \mathbf{h}_{j, j, k}}{1+\widehat{\mathbf{h}}_{j, j, k}^{\mathrm{H}} \mathbf{Q}_{j}^{(k)}\left(\rho \sigma^{2}\right) \widehat{\mathbf{h}}_{j, j, k}} \\
& -\frac{\mathbf{h}_{j, j, k}^{\mathrm{H}} \mathbf{Q}_{j}^{(\mathrm{k})}\left(\rho \sigma^{2}\right) \widehat{\mathbf{h}}_{j, j, k} \widehat{\mathbf{h}}_{j, j, k}^{\mathrm{H}} \mathbf{Q}_{j}^{(k)}\left(\rho \sigma^{2}\right) \widehat{\mathbf{H}}_{j, j_{k}} \overline{\mathbf{D}}_{k} \widehat{\mathbf{H}}_{j, j_{k}}^{\mathrm{H}} \mathbf{Q}_{j}^{(\mathrm{k})}\left(\rho \sigma^{2}\right) \widehat{\mathbf{h}}_{j, j, k} \widehat{\mathbf{h}}_{j, j, k}^{\mathrm{H}} \mathbf{Q}_{j}^{(\mathrm{k})}\left(\rho \sigma^{2}\right) \mathbf{h}_{j, j, k}}{\left(1+\widehat{\mathbf{h}}_{j, j, k}^{\mathrm{H}} \mathbf{Q}_{j}^{(\mathrm{k})}\left(\rho \sigma^{2}\right) \widehat{\mathbf{h}}_{j, j, k}\right)^{2}} \underset{K \rightarrow \infty}{\longrightarrow} 0 .
\end{aligned}
$$

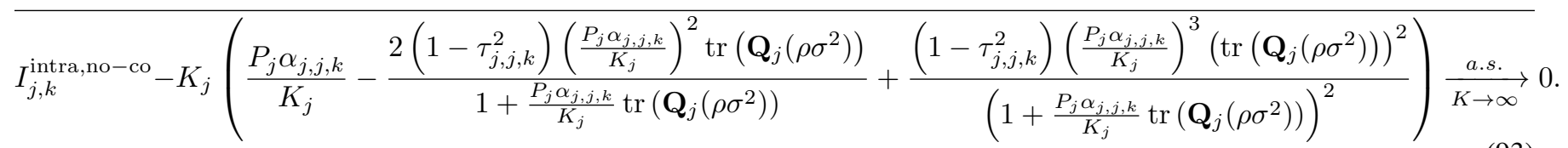

\section{B. Sketch of Proof of Theorem 2}

In this section, we sketch the proof of Theorem 2 as the derivations rely most often on the same arguments as those presented in Theorem's 1 proof above.

Considering the design of the coordinated SLNR-MAX precoding (21), one should note that the beamforming vectors involve now matrix $\widehat{\mathbf{H}}_{j}$ given by:

$$
\widehat{\mathbf{H}}_{j}=\left[\beta_{j} \widehat{\mathbf{H}}_{j, 1}, \beta_{j} \widehat{\mathbf{H}}_{j, 2} \cdots, \widehat{\mathbf{H}}_{j, j}, \cdots, \beta_{j} \widehat{\mathbf{H}}_{j, L}\right] \text {, }
$$

This must be compared to the non-coordinated SLNR-MAX (11) in which the beamforming vectors involve only $\widehat{\mathbf{H}}_{j, j}$. Furthermore, some care must be paid when applying propositions 1 and 2 as the number of columns of matrix $\widehat{\mathbf{H}}_{j}$ differs from the variance scaling $\frac{1}{K_{j}}$. To handle this issue, we need to consider the following relation:

$$
\left(\widehat{\mathbf{H}}_{j} \widehat{\mathbf{H}}_{j}^{\mathrm{H}}+\rho \sigma^{2} \mathbf{I}_{M_{j}}\right)^{-1}=\frac{K_{j}}{K} \mathbf{Q}_{j}\left(\frac{K_{j}}{K} \rho \sigma^{2}\right),
$$

where

$$
\mathbf{Q}_{j}\left(\frac{K_{j}}{K} \rho \sigma^{2}\right)=\left(\frac{K_{j}}{K} \widehat{\mathbf{H}}_{j} \widehat{\mathbf{H}}_{j}^{\mathrm{H}}+\frac{K_{j}}{K} \rho \sigma^{2} \mathbf{I}_{M_{j}}\right)^{-1} .
$$

Considering $e_{j}^{\mathrm{co}}\left(\rho \sigma^{2}\right)$ in (37) and $\tilde{\vartheta}_{j}^{\mathrm{co}}\left(\rho \sigma^{2}\right)$ in (41) and letting $t_{c o}$ be as in (38), a direct application of Propositions 1 and 2 yields:

$$
\frac{1}{K} \operatorname{tr}\left(\mathbf{A}_{M} \mathbf{Q}_{j}\left(t_{c o}\right)\right)-\frac{e_{j}^{\text {no }- \text { co }}\left(t_{c o}\right)}{t_{c o}} \frac{1}{K} \operatorname{tr}\left(\mathbf{A}_{M}\right) \underset{K \rightarrow \infty}{\stackrel{a . s .}{\longrightarrow}} 0,
$$

and

$$
\begin{aligned}
& \frac{1}{K} \operatorname{tr}\left(\mathbf{A}_{M}\left(\mathbf{Q}_{j}\left(t_{c o}\right)\right)^{2}\right) \\
& -\frac{\left(\tilde{e}_{j}^{\mathrm{co}}\left(t_{c o}\right)\right)^{2}}{\left(t_{c o}\right)^{2}\left(1-\frac{1}{\tilde{c}_{j}} \tilde{\vartheta}_{j}^{\mathrm{co}}\left(t_{c o}\right)\right)} \frac{1}{K} \operatorname{tr}\left(\mathbf{A}_{M}\right) \underset{K \rightarrow \infty}{a . s .} 0,
\end{aligned}
$$

where $\mathbf{A}_{M} \in \mathbb{C}^{M_{j} \times M_{j}}$ is an abitrary sequence of matrices with bounded spectral norm.
As mentioned before, the procedure to derive the asymptotic expressions of Theorem 2 is in essence similar to the proof of Theorem 1. In fact, two differences are worthy of mentioning and have to be considered. First, the convergences (97)-(100) have to be taken into account and applied wherever necessary to preserve the coherence with regard to the Random Matrix Theory tools in use. Apart from this fact, the working out of $\bar{S}_{j, k}^{\mathrm{co}}$ and $\bar{I}_{j, k}^{\mathrm{intra} \text {,co }}$ obeys exactly the same arguments as for $\bar{S}_{j, k}^{\text {no-co }}$ and $\bar{I}_{j, k}^{\text {intra,no-co }}$, respectively. The second difference between the two theorems takes place when dealing with the term $I_{j, k}^{\text {inter,co }}$. As explained in section III, when coordination is implemented among base stations, the latter will have knowledge of both intended and non-intended channels which will be used later on in the beaforming. Hence, regardless of the parameter $\beta_{j}$, the inter-cell interference and the intra-cell interference takes conceptually the same form. Therefore, in Theorem 2, the derivation of $\bar{I}_{j, k}^{\text {inter,co }}$ follows the same steps that led to finding $\bar{I}_{j, k}^{\text {intra,no-co }}$. Details are thus omitted.

\section{ACKNOWLEDGMENT}

This publication is based upon work supported by the King Abdullah University of Science and Technology (KAUST) Office of Sponsored Research (OSR).

\section{REFERENCES}

[1] J. Andrews, S. Buzzi, W. Choi, S. Hanly, A. Lozano, A. Soong, and J. Zhang, "What will 5G be?" IEEE Journal on Selected Areas in Communications, vol. 32, no. 6, pp. 1065-1082, June 2014.

[2] M. Kountouris and N. Pappas, "Hetnets and massive MIMO: Modeling, potential gains, and performance analysis," in IEEE-APS Conference on Antennas and Propagation in Wireless Communications (APWC) Topical, Sept 2013, pp. 1319-1322.

[3] X. Ge, S. Tu, G. Mao, C. X. Wang, and T. Han, "5G ultra-dense cellular networks," IEEE Wireless Communications, vol. 23, no. 1, pp. 72-79, February 2016.

[4] N. Bhushan, J. Li, D. Malladi, R. Gilmore, D. Brenner, A. Damnjanovic, R. T. Sukhavasi, C. Patel, and S. Geirhofer, "Network densification: the dominant theme for wireless evolution into 5G," IEEE Communications Magazine, vol. 52, no. 2, pp. 82-89, February 2014.

[5] N. Wang, E. Hossain, and V. K. Bhargava, "Joint downlink cell association and bandwidth allocation for wireless backhauling in two-tier hetnets with large-scale antenna arrays," IEEE Transactions on Wireless Communications, vol. 15, no. 5, pp. 3251-3268, May 2016. 
[6] E. Hossain, M. Rasti, H. Tabassum, and A. Abdelnasser, "Evolution toward 5G multi-tier cellular wireless networks: An interference management perspective," IEEE Wireless Communications, vol. 21, no. 3, pp. 118-127, June 2014

[7] J. Hoydis, M. Kobayashi, and M. Debbah, "Green small-cell networks," IEEE, Vehicular Technology Magazine, vol. 6, no. 1, pp. 37-43, March 2011.

[8] D. Lopez-Perez, I. Guvenc, G. de la Roche, M. Kountouris, T. Quek, and J. Zhang, "Enhanced intercell interference coordination challenges in heterogeneous networks," IEEE Wireless Communications, vol. 18, no. 3, pp. 22-30, June 2011.

[9] L. Sanguinetti, A. L. Moustakas, and M. Debbah, "Interference management in $5 \mathrm{~g}$ reverse tdd hetnets with wireless backhaul: A large system analysis," IEEE Journal on Selected Areas in Communications, vol. 33, no. 6, pp. 1187-1200, June 2015.

[10] Y. Huang, S. He, S. Jin, and W. Chen, "Decentralized energy-efficient coordinated beamforming for multicell systems," IEEE Transactions on Vehicular Technology, vol. 63, no. 9, pp. 4302-4314, Nov 2014.

[11] S. Lakshminarayana, M. Assaad, and M. Debbah, "Coordinated multicell beamforming for massive mimo: A random matrix approach," IEEE Transactions on Information Theory, vol. 61, no. 6, pp. 3387-3412, June 2015.

[12] F. Zhang, Y. Huang, S. Jin, L. Jiang, and G. Wang, "Reduced-backhaul coordinated beamforming for massive mimo heterogeneous networks," in 2015 IEEE Wireless Communications and Networking Conference (WCNC), March 2015, pp. 129-134.

[13] R. Zakhour and S. V. Hanly, "Base station cooperation on the downlink: Large system analysis," IEEE Transactions on Information Theory, vol. 58, no. 4, pp. 2079-2106, April 2012.

[14] J. Mirza, P. Smith, and P. Dmochowski, "Coordinated regularized zeroforcing precoding for multicell MISO systems with limited feedback," IEEE Transactions on Vehicular Technology, vol. PP, no. 99, pp. 1-1, 2016.

[15] P. Bhat, S. Nagata, L. Campoy, I. Berberana, T. Derham, G. Liu, X. Shen, P. Zong, and J. Yang, "LTE-advanced: an operator perspective," IEEE Communications Magazine, vol. 50, no. 2, pp. 104-114, February 2012.

[16] R. Irmer, H. Droste, P. Marsch, M. Grieger, G. Fettweis, S. Brueck, H. P. Mayer, L. Thiele, and V. Jungnickel, "Coordinated multipoint: Concepts, performance, and field trial results," IEEE Communications Magazine, vol. 49, no. 2, pp. 102-111, February 2011.

[17] H. Dahrouj and W. Yu, "Coordinated beamforming for the multi-cell multiantenna wireless system," IEEE Transactions on Wireless Communications, vol. 9, no. 5, pp. 1748-1759, May 2010.

[18] E. Bjornson and E. Jorswieck, "Optimal resource allocation in coordinated multi-cell systems," Foundations and Trends in Communications and Information Theory, vol. 9, no. 2-3, pp. 113-381, 2013.

[19] M. Sadek, A. Tarighat, and A. Sayed, "A leakage-based precoding scheme for downlink multi-user MIMO channels," IEEE Transactions on Wireless Communications, vol. 6, no. 5, pp. 1711-1721, May 2007.

[20] S. Feng, M. M. Wang, W. Yaxi, F. Haiquang, and L. Jinhui, "An efficient power allocation scheme for leakage-based precoding in multi-cell multiuser MIMO downlink," IEEE Communications Letters, vol. 15, no. 11, Oct. 2011.

[21] P. Cheng, M. Tao, and W. Zhang, "A new slnr-based linear precoding for downlink multi-user multi-stream mimo systems," IEEE Communications Letters, vol. 14, no. 11, pp. 1008-1010, November 2010.

[22] S. Wang, Y. Yu, C. Zhai, W. Zhang, W. Wang, and H. Wang, "An mmse based signal to leakage plus noise ratio precoding scheme with other cell interference," in IEEE 78th Vehicular Technology Conference (VTC Fall), Sept 2013.

[23] P. Patcharamaneepakorn, S. Armour, and A. Doufexi, "On the equivalence between slnr and mmse precoding schemes with single-antenna receivers," IEEE Communications Letters, vol. 16, no. 7, Jul. 2012.

[24] H. Tataria, M. Shafi, P. Smith, and P. Dmochowski, "Coordinated two-tier heterogeneous cellular networks with leakage based beamforming," 2015. [Online]. Available: arXiv:1503.01566

[25] S. Wagner, R. Couillet, M. Debbah, and D. T. M. Slock, "Large system analysis of linear precoding in correlated MISO broadcast channels under limited feedback,' IEEE Transactions on Information Theory, vol. 58, no. 7, pp. 4509-4537, July 2012.

[26] J. Hoydis, S. ten Brink, and M. Debbah, "Massive MIMO in the UL/DL of cellular networks: How many antennas do we need?" IEEE Journal on Selected Areas in Communications, vol. 31, no. 2, pp. 160-171, February 2013.

[27] L. Sanguinetti, R. Couillet, and M. Debbah, "Large system analysis of base station cooperation for power minimization," IEEE Trans.
Wireless Communications, vol. 15, no. 8, pp. 5480-5496, 2016. [Online] Available: http://dx.doi.org/10.1109/TWC.2016.2560178

[28] P. Billingsley, Probability and Measure, 3rd ed. New York: Wiley, 1995

[29] M. Gudmundson, "Correlation model for shadow fading in mobile radio systems," Electronics Letters, vol. 27, no. 23, pp. 2145-2146, Nov 1991.

[30] B. Golkar and E. Sousa, "A network shadow fading model for autonomous infrastructure wireless networks," in Proceedings of the 20th European Signal Processing Conference (EUSIPCO), Aug 2012, pp. 2659-2663.

[31] R. B. Dozier and J. W. Silverstein, "On the empirical distribution of eigenvalues of large dimensional information-plus-noise-type matrices," Journal of Multivariate Analysis, vol. 98, no. 4, pp. 678 - 694, 2007.

[32] D. Paul and J. W. Silverstein, "No eigenvalues outside the support of the limiting empirical spectral distribution of a separable covariance matrix," Journal of Multivariate Analysis, vol. 100, no. 1, pp. 37 - 57, 2009.

[33] R. Couillet and M. Debbah, Random Matrix Methods for Wireless Communications. Cambridge, New York: Cambridge University Press, 2011.

[34] W. Hachem, O. Khorunzhiy, P. Loubaton, J. Najim, and L. Pastur, "A new appraoch for mutual information analysis of large dimensional multiantenna channels," IEEE Transactions on information theory, vol. 54, no. 9, pp. 3987-4004, 2008.

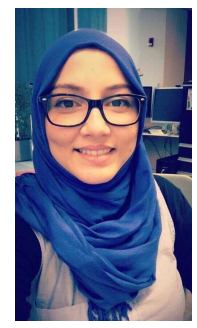

Ikram Boukhedimi was born in Blida, Algeria She received the Electrical Engineering degree from the École Polytechnique d'Alger, Algeria, in 2013 and a M.S. in Mathematics, Control and Electrical Engineering from Université de Poitiers, France in 2014. Currently, she is working towards the Ph.D in Electrical Engineering at King Abdullah University of Science and Technology, Saudi Arabia. Her research interests include performance analysis and random matrix theory approaches applied to wireless communication systems.

Abla Kammoun was born in Sfax, Tunisia. She received the engineering degree in signal and systems from the Tunisia Polytechnic School, La Marsa, and the Master's degree and the Ph.D. degree in digital communications from Télécom Paris Tech [then École Nationale Supérieure des Télécommunications (ENST)]. From June 2010 to April 2012, she has been a Postdoctoral Researcher in the TSI Department, Télécom Paris Tech. Then she has been at Supèlec at the Alcatel-Lucent Chair on Flexible Radio until December 2013. Currently, she is a research scientist at KAUST university. Her research interests include performance analysis, random matrix theory, and semi-blind channel estimation. She is the recipient of the SAM 2014 second Prize Best paper award.

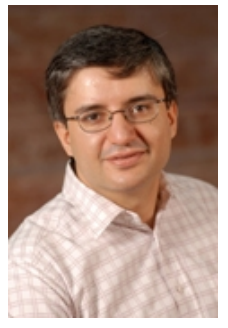

Mohamed-Slim Alouini (S'94, M'98, SM'03, F09) was born in Tunis, Tunisia. He received the Ph.D. degree in Electrical Engineering from the California Institute of Technology (Caltech), Pasadena, CA, USA, in 1998. He served as a faculty member in the University of Minnesota, Minneapolis, MN, USA, then in the Texas A\& M University at Qatar, Education City, Doha, Qatar before joining King Abdullah University of Science and Technology (KAUST), Thuwal, Makkah Province, Saudi Arabia as a Professor of Electrical Engineering in 2009. His current research interests include the modeling, design, and performance analysis of wireless communication systems. 